\title{
Slow Infusion of Information Technology in Master Bank of Pakistan - A Case Study
}

\author{
${ }^{*}$ Fareeha Khalil1 ${ }^{1}$, HammadUsmani2 ${ }^{2}$ Muhammad Farhan ${ }^{1}$ \\ ${ }^{1}$ Hailey College of Commerce, University of the Punjab, Lahore, Pakistan \\ 2The University of Lahore, Pakistan \\ *farihakhalil@hotmail.com
}

\begin{abstract}
E-Banking is now a well know phenomenon in the industry and amongst the banking users in Pakistan. This paper addresses the issues encountered and lessons derived by largest Pakistani bank while developing its e-banking capabilities. The paper explores some burning issues hindering key decisions of shifting to e-banking, e-banking delivery channel choice, enticing customers, and managing e-channel conflict to be made by bank. The findings indicated that the obstacles related to e banking customer location, customer satisfaction and the Bank's software's and communication infrastructure capabilities are influential factors in motivating the decision for shifting to a high-tech bank. The bank's E-channel choice is influenced by competence of application and software technologies that the bank acquires and the nature of diffusion of information and communications among its stakeholders. Technological development in Banking practices and awareness in customers due to presence of foreign and domestic banks in Pakistani financial market influenced the Master Bank to switch over to complete automated banking system. The sophistication of ebanking services and the further rapid improvement appears to influence strategies to entice E-channel conflict issues. The paper concludes by suggesting the Bank to optimally utilize its resources by deploying efficient applications and software's and third party alliances and partnerships required to offer customized technology enabled banking services and develop appropriate e-banking risk mitigation strategies that bring the bank into a win-win position.
\end{abstract}

\section{Keywords: Information Technology, Banking, Pakistan, E-Banking, Software}

\section{Introduction}

The information system has three ingredients. Firstly the essential tools (Hardware) as property, secondly the information enclosed in the structure (software) and finally the terminology that enables the user to get access of this information (Instructions); so this information management joins the business management with computer sciences, we live in the era of advanced technology and we find that many business organizations are totally reliant on the information technology in its business administration, because of the good use of information technology all the stake holders get benefits from it as this information and communication system saves an organization's time, efforts and money (AL-Rawashdeh, Abu-Errub, Areiqat, \& Dbbaghieh, 2012). The information technology has brought revolution in the banking industry through Ebanking, E-payments, security investments and information exchange (Berger, 2003). Now banks can provide more assorted services to its customers with less human capital, by looking at this pattern its seems that information technology can equally influence the profitability of the banking industry (Ho \& Mallick, 2010).

Strategic Importance of IT in organizational Decision Making: Information technology is playing a vital role in driving development and growth, promoting modernization and developing competitavness; it is need of the era to infuse the latest technology in any envioment to compete with the increasing global enviorment and information technology has become a dominantinstrument when it is used as a part of development stratagemunited with partnerships between governments, business and civil society (World Bank, 2003). The role of information technology is vital in the development of any economy across the world, and as far as banking industry is concerned it is one of the very first to implement information technology back in 1960s and has developed itseld by infusing the information technology. The information technology has proved itself as a strategic resource for organizations in today's global economy. Now, the information technology is being regarded as most important factor when it is compared to capital and human resource. Some experts believed that the labour was the most critical factor in agricultural age, Capital in industrial age and Information in post-industrial age. Managing information effectively is now being most challenging job in 
business environment. Many believe that the way organizations manage their information will increasingly determine their survival and growth in future (AL-Rawashdeh at el, 2012). The banking industry is being selected here for study because as a part of the service industry it is supposed to have one of the lowest IT productivity (Shu and Strassman, 2005).

Role of Information Technology in Banking Industry: Information technology has transformed the way the organizations do business especially the Banking industry. Manual banking functions have been automated with the development of personal computers, business software and Internet-based intranets. While many of Banks in Pakistan have developed internal technology to increase the effectiveness of their business functions, they have also introduced many banking products with extensive use of information technology to enhance operational efficiency and to achieve strategic goals. Developing different uses for information technology and various computer-based products are helping the banks in gaining a competitive advantage. The usage of information technology (IT), broadly referring to computers and peripheral equipment, has seen tremendous growth in service industries in the recent past. The most obvious example is perhaps the banking industry, where through the introduction of IT related products in internet banking, electronic payments, security investments, information exchanges (Berger, 2003). As far as the relationship of information technology and bank's performance is concerned we find positive relationship through literature; firstly with the infusion of information technology a bank can reduce its operational costs i.e. low value-added business transactions can be handled through internet (e.g. bank statements, balance inquiries, funds transfer) while focusing on the high value-added business transactions(e.g. investment banking, deposit mobilization, rational lending)(Ho \& Mallick, 2010,Farrell and Saloner, 1985; Katz and Shapiro, 1985; Economides and Salop, 1992). There is also a positive relationship between the infusion of information technology and bank's profitability (Kozak, 2005).

Information Technology in Banking Industry of Pakistan: In recent decades, the technological innovations have continued inroads into Pakistan are banking Industry. Information technology has become a pivot factor for managing banking operations in terms of both products and services. It has also become key driver for financial inclusion in terms of all banking operations and products. The banking customers who were only exposed to ATMs now enjoy telephone and PC banking, Internet Banking, IVR systems, POS, and multiple hosts of transactions under the umbrella of electronic financial services. The performance of banks is Pakistan is now judged significantly in terms of use of information technology and the definition of customer service standards has been changed due to heavy reliance on information technology. The information technology platform has enhanced business volumes of Pakistani banks and unbanked geographical regions have also been captured. Many of private sector Pakistani banks have introduced branchless banking and the banking customers now do not need to visit the branches here they are maintaining their accounts. Many private sector and foreign Banks are moving towards very sophisticated information technology based solutions to facilitate financial services. Further, some of private and foreign banks have switched to Core Banking Solutions showing a paradigm shift in banking practices and operations.

Despite having magnificent growth of IT based banking solutions, Pakistani banks have to go long way in considerable implementation of the information technology as Pakistan's banking system is still far from desirable levels of quantity and quality of IT based banking solutions. Pakistani banks still have huge space to excel as $85 \%$ of the population of Pakistan is still unbanked and untapped. Only some of foreign and private sector banks have made remarkable progress in developing e-banking platforms, the huge public sector banks despite of having huge network and depositor base like Master Bank yet have to start from scratch to establish on overall basis a sound information technology based system to support both internal MIS and efficient customer services. The core issue this paper addresses is the need for infusion of information technology in Banking Practices in public sector Pakistani banks. Also, the challenges a bank may expose to during the process of full IT adoption and possible solutions/recommendations to cater those challenges. The paper begins with a brief literature review on importance of Information Technology in banking, which is followed by the case of a large public sector Pakistani bank, and the analysis of its key factors vital in adoption of Information Technology for strategic goal accomplishment. It concludes with a set of recommendations for bank under discussion. 


\section{Literature Review}

This is an era if competition as far as banking is concerned; settings up of excellent business operational tactics are necessary for survival; within extremely volatile and unpredictable financial market it depends on banks how they utilize the information technology to maintain the client loyalty, maximize the profit, reduce cost and maintain a sustainable growth rate; as competition is not only limited to geographical limits because large multinational banking organizations make it difficult for small ones to survive so without an valuable weapon survival is not possible, Information technology can be that weapon of survival for the banking organizations (Sun, 2010).

Information Technology and Cost \& Profit Efficiency of Banking Sector: The Information Technology has become a crucial enabler for information and knowledge management in organizations (AL-Rawashdeh at el.,2012, Stratigos, 2001; Higgins, 2001,). Kozak (2005) examined the relationship between the progress in IT and profit and cost efficiencies in the US banking sector during 1992-2003. The research revealed that levels of implemented IT and both profitability and cost savings were positively correlated. As far as human capital is concerned no one can deny its importance but it is very difficult to retain this therefore most of the business organizations (banks) are focusing on the available information systems to improve service quality, enhance human capital, improve efficiency, reinvent business structure and develop strategies to get competitive advantage over the others (Liao, Landry Jr, \& Cheung, 2003). By using the information system a bank can also reduce its sunk costs of entering the new markets (Corvoisier \& Gropp, 2009). Al-Otaibi (1992) studied moderating impact of different IT factors on the relationship between strategic decisions and organizational performance. A number of these factors found significantly related to organizational performance.

The strategic decisions regarding the enhanced communication capability through Information Technology facilitate learning from strategic initiatives and the innovation across industries is due to use of IT (Andersen, 2001). Reviewing various studies, Urgo (1996) concluded that the investment in IT has positive correlation to the productivity. Aladwani $(2000,2001)$ emphasized that the information system project characteristics and performance have strong relationship. On the other hand, Zhu et al. (2004) studied banking industry to ascertain relationship between advanced computer operating systems, wide and local area networks and internet on Banks' performance. They proved that network systems do not impact the return on assets. The findings of studies described above have provided some evidence of variation in impact of IT in different organizations merely due to differences in organizational characteristics, the usefulness of these studies is limited to organization's reliance on IT investment and firm performance (Dos Santos and Pfeffers, 1993).

Information Technology and Satisfaction of Banking Customer: The satisfaction of Customer has become focus of attention of managers in various sectors (retailing, e-government, telecommunications and health services, etc (Riquelme at el. 2009; Terblanche, 2006; Perreault et al., 2006; Cohen, 2006;Welch et al., 2005; Athanassopoulos and Iliakopoulos, 2003). Szymankski and Henard, 2001 discussed that main reasons for high attention towards customer satisfaction are the positive relation of customer satisfaction with market share, customer retention, customer loyalty, business extension and higher profits. In a study, (Chothani et al. 2004) concluded that $5 \%$ increase in customer retention increases profitability by $35 \%$ in banking business, for this, banks are more inclined towards retaining customers for increasing their market share. Only those banks are likely to survive and grow in new millennium that re-organize whole of their payment and delivery systems and apply IT to their operations. Further, the banks must re-structure their services in order to properly position them within the framework of the dynamism of information technology and communication systems (Corvoisier\& Gropp, 2009; Woherem, 2000). Jayawardhena and Foley (2000) stated that banks in West European countries had pioneered internet-based banking services. They found that a positive relationship between user satisfaction and the multimedia content and hypertext connection of websites. Joseph and Stone (2003) stated that adoption of intensive telecommunication technologies induces more customers to use self-service delivery systems. The innovations in IT are strategic tools for enhancing the value of customer relationship. They help in reducing the costs of financial transactions, improving the allocation of financial resources, increasing customer satisfaction and increasing the competitiveness and efficiency of financial institutions (Subbarao, 2009). Riquelme at el. (2009) discussed in their study that 
customers using internet banking experienced highest levels of satisfaction in terms of accessibility of eservices.

Strategic Importance of Information Technology in Organizations: Information technology is increasingly being taken by management as an opportunity to leverage sustainable competitive advantage (SCA) in organizations (Remenyi 1991). IT has become one of the most powerful strategic tools as the managers must have to create sustainable comparative advantage from IT, although, it is not simple (Domegan, 1995). Burn and Loch (2001) stated that organizational, sociological and cultural factors might inhibit effective transformation to global application of IT caused by the inclusion of internet and other telecommunication systems. Chou and Jou (1999) and Dasgupta, (1999) studied Asian region and found that industrial settings caused significant differences in the perceptions of information systems' managers. They concluded that organizational climate, culture, size, environmental factors, government policies, and market forces are main influential factors on the perceptions of managers and their strategic decision-making in Taiwanese and Indian firms. In the Middle East, the adoption of IT was slow due to political, religious, cultural and ethical concerns (Al-Tawil, 2001; Houissa, 2000). Lin et al. (2005) presented an eChain bank accounting system integrating value chain product and emphasized that value chain activities like account activities and fund capitalization must be coupled together. The implementation of this system will ensure increased customer satisfaction together with sustainable comparative advantage. Ju et al. (2005) through a strategic contingency model for technology alliance empirically found that organizations with higher IT absorption tendency, higher risk evasiveness, higher R\&D orientation and higher expertise of top management tend to gain higher competitive advantages.

Westerback (1999) reviewed public agencies and found that strategic information technology practices cause improved service delivery and better returns on investment. Also, the role of human resource in planning and management of IT applications has changed a great deal and recent studies have focused that new sets of skills, strategies related to their employment, development and retention are needed (Burn et al., 1995; Gordon, 2002; Hallam, 2001; Harwood, 2001; Heckman, 1998; Slowinsky, 2000; Smith and Webster, 2000). In the financial services industry, product and services creation is a result of a chain of complex activities of a team of human resource. Further, in the banking industry, profit related objectives are being preferred over volume based sales with intensive use of IT (Mabberly, 1996). Harold and Jeff (1995) empirically proved that financial service providers should restructure their traditional delivery systems to remain viable. They claimed the failure on the part of senior management in banks to understand the importance of Technology enable delivery systems as the most significant shortcoming in the banking industry.

E-Banking Infrastructure in Pakistan: Initially internet banking was launched in Pakistan to provide a limited number of services and it was sort of hype in the market. In the past years there has been greater understanding and development towards various services being offered by banks through internet banking. Customer readiness, acceptance \& security awareness is also a factor which is improving as usage of internet banking is increasing. Banks have also begun to understand the cost \& benefits of internet banking.In Pakistan, there was also an internet bandwidth issue which has since been resolved by the availability of better internet network availability. Utility companies are also gearing up for offering bill payment facilities through various electronic channels including Internet Banking. Table 1 is showing the growth of technology enabled banking transactions in Pakistan over years 2006 to 2010 as follows;

Table1: E-Banking Infrastructure in Pakistan

\begin{tabular}{|c|c|c|c|c|c|c|c|}
\hline Items & UNIT & 2006 & 2007 & 2008 & 2009 & 2010 & Growth \\
\hline \multicolumn{8}{|c|}{ E BANKING INFRASTRUCTURE } \\
\hline $\begin{array}{l}\text { Real Time Online } \\
\text { Branches (RTOB) }\end{array}$ & Number & 3555 & 4179 & 5282 & 6040 & 6671 & $87.65 \%$ \\
\hline $\begin{array}{l}\text { Automated Teller } \\
\text { Machines (ATM) }\end{array}$ & Number & 1612 & 2294 & 3121 & 3999 & 4465 & $176.98 \%$ \\
\hline Point of Sale (POS) & Number & 32331 & 46329 & 43903 & 49715 & 52049 & $60.99 \%$ \\
\hline \multicolumn{8}{|c|}{ CREDIT CARD TRANSACTIONS } \\
\hline Credit Cards & Thousands & 973 & 1698 & 1777 & 1664 & 1613 & $65.77 \%$ \\
\hline Outstanding Amount & Million Rs. & 33538 & 42822 & 44427 & 35533 & 28280 & $-15.68 \%$ \\
\hline
\end{tabular}




\begin{tabular}{|c|c|c|c|c|c|c|c|}
\hline DEBIT CARDS & Thousands & 2740 & 3983 & 4905 & 6395 & 8140 & $197.08 \%$ \\
\hline \multicolumn{8}{|c|}{ E BANKING TRANSACTIONS } \\
\hline No. of Transactions & Thousands & 67774 & 99367 & 124447 & 159783 & 196301 & $189.64 \%$ \\
\hline Value of Transactions & Billion Rs. & 7040 & 10497 & 13893 & 14373 & 17333 & $146.21 \%$ \\
\hline \multicolumn{8}{|l|}{ ATM TRANSACTIONS } \\
\hline No. of Transactions & Thousands & 35025 & 51511 & 67912 & 91126 & 115677 & $230.27 \%$ \\
\hline Value of Transactions & Billion Rs. & 211 & 316 & 453 & 669 & 905 & $328.91 \%$ \\
\hline \multicolumn{8}{|l|}{ POS TRANSACTIONS } \\
\hline No. of Transactions & Thousands & 10896 & 15589 & 17485 & 18280 & 15677 & $43.88 \%$ \\
\hline Value of Transactions & Billion Rs. & 32 & 54 & 69 & 90 & 75 & $134.37 \%$ \\
\hline \multicolumn{8}{|l|}{ RTOB TRANSACTIONS } \\
\hline No. of Transactions & Thousands & 21018 & 30731 & 36855 & 47279 & 60615 & $188.39 \%$ \\
\hline Value of Transactions & Billion Rs. & 6774 & 10090 & 133317 & 13538 & 16202 & $139.18 \%$ \\
\hline \multicolumn{8}{|c|}{ OTHER BANKING CHANNELS (Internet Banking, Mobile Banking, Call Centers etc) } \\
\hline No. of Transactions & Thousands & 835 & 1537 & 2195 & 3098 & 4336 & $419.28 \%$ \\
\hline Value of Transactions & Billion Rs. & 23 & 37 & 54 & 77 & 150 & $552.17 \%$ \\
\hline
\end{tabular}

The figures indicated tremendous growth of IT enabled products and transaction from year 2006 to 2010 especially the Banking channels like Internet Banking, Call Centers and Mobile Banking has increased by $552.17 \%$ showing the seriousness of senior management of Banks in Pakistan in understanding the profitability potential, time saving and provision of customer oriented services by adopting IT and Technology enabled products.

\section{Methodology}

The case study approach is being used for this study to correlate the banking issues with real life. Yin (1994) emphasized on use of case study where specific research questions like "why" or "how" are to be addresses. By asking how or why, the case study approach valuably contributes towards generating knowledge or construction of a new perspective of the phenomena which is to be investigated (Boateng and Molla, 2006,Eisenhardt, 1989). Within this research, the objective is to ascertainthe factors responsible for slow induction of Information technology in banking practices in Master Bank. Also, the elements that influence this decisions and the lessons that can be derived out of this study. The bank used in this research is a largest Pakistani bank, which for the sake of confidentiality would be referred to as Master Bank. Data and information were gathered through interviews with the IT department staff at both Head Office and local area office level. Additional data were collected by analysis of Bank's website and director's report to shareholders. The current IT applications being used by the Bank were also overviewed. The IT manuals issued by Bank from time to time were studied as well.

\section{The Master Bank}

Master Bank is the largest commercial bank operating in Pakistan. Its balance sheet size surpasses that of any of the other banks functioning locally. It has redefined its role and has moved from a public sector organization into a pure commercial bank. Year 2010 was a milestone year for the bank as it has become the first financial institution in the country to surpass the One Trillion Rupee' mark. Total assets of the bank were at Rs.1.035 trillion at the year end, up by $9.6 \%$ from year end 2009, an appreciable growth in a challenging economic environment. Pre- tax profit increased by $15 \%$ from Rs. 21.3 billion to Rs. 24.4 billion. The increase is owing to higher core revenues and lower provision charge. Net interest income increased by $15 \%$ from last year, while fee income was up by $8 \%$ on account of higher trade finance and general banking income. After tax profit, however, remained at last year level of Rs.17.6 billion due to prior year's tax reversal of Rs.4.1 billion in 2009 (Directors' Report to the shareholders, 2010). The Bank's services are available to individuals, corporate entities and government. While it continues to act as trustee of public funds and as the agent to the State Bank of Pakistan (in places where SBP does not have a presence) it has diversified its business portfolio and is today a major lead player in the debt equity market, corporate investment banking, retail and consumer banking, agricultural financing, treasury services and is showing growing interest in promoting and developing the country's small and medium enterprises and at the same time fulfilling its 
social responsibilities, as a corporate citizen. According to consolidated financial statements of year 2010, the bank has declared its deposits as PKR 832,134 million.

Evolution of Information Technology In Master Bank: The Master Bank faces slow growth of Information technology for its management information system and customer products. The table 2 shows year wise progress of IT in Master Bank;

\section{Table 2: Evolution of Information Technology In Master Bank}

\begin{tabular}{|c|c|}
\hline Year & DESCRIPTION \\
\hline 1949 & Master Bank Licensed \\
\hline 1983 & Launched first Online Branch \\
\hline 1996 & First ATM installed \\
\hline 1997 & Credit Cards introduced \\
\hline 1999 & $\begin{array}{l}\text { Branch Back Office System introduced (A decentralized, } \\
\text { stand alone multi user system for Branch operations) }\end{array}$ \\
\hline 2002 & $\begin{array}{l}\text { Electronic Banking System Introduced (A centralized and } \\
\text { real time online system) }\end{array}$ \\
\hline 2003 & First static website launched \\
\hline 2005 & $\begin{array}{l}\text { Web Based Remittance from USA (A web based application } \\
\text { for home remittances) }\end{array}$ \\
\hline 2006 & Up gradation of Website into partially dynamic \\
\hline 2007 & Call Center for retail products \\
\hline 2009 & $\begin{array}{l}\text { Web Based Remittance from KSA / UAE } \\
\text { Full Fledge dynamic Website Launched }\end{array}$ \\
\hline 2010 & First Branch converted into Core Banking Branch \\
\hline
\end{tabular}

A very steady IT infusion is evident from the facts narrated in table 6.1.The Master Bank took more than 40 years to understand the utility of being online when it launched its first online branch in 1983. The advancement in Technology by adopting a real time online electronic Banking contributed little towards operational efficiency as the Master Bank has only 384 branches working as online out of its 1256 domestic branches. In last decade the Master Bank stepped into a rapid adoption of IT and technology based products by launching dynamic website and converting its first Branch using Core banking Application. The Master Bank of Pakistan is currently working mainly with ATM / debit cards and web based remittances application, conceived while comparing E Banking infrastructure in domestic and foreign banks operating in Pakistan with Master Bank.

Table 3: E Banking Channel

\begin{tabular}{|c|c|c|c|}
\hline E Banking Channel & $\begin{array}{l}\text { Domestic \& Foreign Banks } \\
\text { in Pakistan (number) }\end{array}$ & $\begin{array}{l}\text { Master Bank } \\
\text { (number) }\end{array}$ & $\begin{array}{l}\text { Share of Master Bank in } \\
\text { Market (\%) }\end{array}$ \\
\hline ATM & 4465 & 239 & 5.35 \\
\hline Debit Cards & $8,140,000$ & 550,000 & 6.75 \\
\hline Credit Cards & 973,000 & -- & -- \\
\hline POS & 52,049 & -- & -- \\
\hline banking & $4,336,000$ & 804,000 & 18.54 \\
\hline
\end{tabular}

Table 4 shows that the Master Bank stands far behind foreign and domestic Banks operating in Pakistan in offering technology enabled and electronic Banking products to its customers, heavily relying on issuance of ATM debit cards. However, its two Web based remittance products for transfer of funds from USA to Pakistan and from KSA \& UAE to Pakistan have made the Master Bank the market leader by capturing $18.54 \%$ of total home remittances inflowing in Pakistan. To find out the level of infusion of Information Technology in Master Bank, the Diniz model (1998) is being used here which delineates three functional areas and three levels of activity in table 4 : 
Table 4: Information Technology in Master Bank

\begin{tabular}{llll}
\hline & BASIC & INTERMEDIARY & ADVANCED \\
\hline \multirow{4}{*}{ Information Delivery } & Electronic Brochure & Search Engine & Subscription \\
& News & Reports download & Advertisement \\
& Means of Contact & Recruitment forms & Discussion group \\
\multirow{2}{*}{ Transactional } & Special Events & Hot links & Interface Customization \\
Channels & Cheque Book requests & Balance Enquiry & Electronic Cash \\
& Card Requests & Bill Payments & Electronic Signature \\
Customer & E-mail & Investment Advisor & Video Conference \\
Relationship & Suggestion forms & Calculations & Chart \\
& Complain forms & Software Download & Services Development \\
\hline
\end{tabular}

From Diniz Model (1998) it is revealed that Master Bank is partially in the informational stage of induction of IT where its functions are limited to a website and partially in its transactional phase where the balance Inquiry and electronic signature and launch of first Core Banking branch.

\section{Master Banks' Banking Operation Applications and Software's}

In the public sector, the changing competitive environment has spurred the banking organizations to go for delivering greater focus on quality of services and more flexibility, curtailing costs at the same time. The Central Bank is encouraging public sector banking organizations to adopt new IT based solutions and systems in order to improve services and lowering operating costs. However in Pakistan, it is evident that the adoption of information technology varies widely across different banks and banks are not adopting IT based techniques despite their apparent benefits. Especially public sector banks, where adoption of information technology tends to be fragmentary and slow. As the banks increase in size, they need to optimize their structure for which increased use of information technology is unavoidable. The public sector banks are characterized by sobering performance, bureaucratization, increased size, greater use of written directives and larger number of non-production personnel. As discussed above, being a public sector bank, the Master Bank has bureaucratic culture and the structural optimization is not heavily relied upon. The top management is focusing more on customer satisfaction and customer loyalty without using information technology unlike many private sector and foreign banks operating in market. Having competitive advantage in terms of its public appearance and wide depositor base, the top management is not giving due weight age and importance to make the Master Bank a cyber-financial super market.

During last decade, the Master Bank has developed a full fledge IT wing at head office and regional level providing back office IT support to the whole network of Master Bank. The IT development division of Master Bank has developed many software applications that are being used in Master Bank like Branch Back Office, Signature Verification System, E-remittance system, Deposit Receipt etc. Currently, Master Bank has 83 Banking operations and Information system applications and softwares, both in house and outsourced many of which are non-operational. The details of the applications and softwares are given in Table7.1. Master Bank has no main software for Banking Operations and retrieval of different reports rather they are working with many stand alone softwares having limited functional utilities. Only one major Electronic Banking System is operational with different online banking modules. Bank is relying on standalone PC based applications and softwares for single users. Also, the bank seems reluctant in outsourcing its information technology development process and making useful $3^{\text {rd }}$ party alliances. Most of the softwares are developed by using Fox pro which is an obsolete programming language and only 5 of its applications are oracle bas. Table 5 shows the details of various softwares, their utilities, database engine and automation level being used in Master Bank and highlights need for a proper centralized information system. 
Table 5: Proper centralized information system

\begin{tabular}{|c|c|c|c|c|c|c|c|c|c|c|}
\hline $\begin{array}{l}\text { S/ } \\
\text { N }\end{array}$ & Application Name & Utilities & $\begin{array}{l}\text { Centralized/ } \\
\text { Decentralize } \\
\text { d }\end{array}$ & 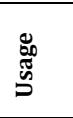 & $\begin{array}{l}\text { In- } \\
\text { house } \\
\text { /3rd } \\
\text { Party }\end{array}$ & $\begin{array}{l}\text { Programming } \\
\text { Languages }\end{array}$ & $\begin{array}{l}\text { Databas } \\
\text { e Engine }\end{array}$ & $\begin{array}{l}\text { System } \\
\text { Platform }\end{array}$ & $\begin{array}{l}\text { Automati } \\
\text { on Level }\end{array}$ & $\stackrel{\mathscr{E}}{\tilde{E}}$ \\
\hline 1 & $\begin{array}{l}\text { Electronic Banking } \\
\text { Systems } \\
\text { (On-line) }\end{array}$ & $\begin{array}{l}\text { Banking } \\
\text { operations for } \\
\text { Online } \\
\text { Branches }\end{array}$ & Centralized & $\begin{array}{l}\text { Multi } \\
\text { user }\end{array}$ & $\begin{array}{l}\text { In } \\
\text { House }\end{array}$ & $\begin{array}{lr}\text { ILE robol, } \\
\text { Cobol, CLP, Java }\end{array}$ & DB-2 & $\begin{array}{l}\text { OS400/ } \\
\text { AS400 }\end{array}$ & On Line & Live \\
\hline \multirow[t]{13}{*}{2} & Branch Back Office & $\begin{array}{l}\text { PC Based } \\
\text { Branch } \\
\text { Banking } \\
\text { operations and } \\
\text { Activities }\end{array}$ & $\begin{array}{l}\text { Decentralize } \\
\mathrm{d}\end{array}$ & $\begin{array}{l}\text { Multi } \\
\text { User }\end{array}$ & $\begin{array}{l}\text { In } \\
\text { House }\end{array}$ & FoxPro 2.6 & $\begin{array}{l}\text { FoxPro } \\
2.6\end{array}$ & DOS & $\begin{array}{l}\text { Stand } \\
\text { Alone }\end{array}$ & Live \\
\hline & $\begin{array}{l}\text { Consumer Loan } \\
\text { Software }\end{array}$ & $\begin{array}{l}\text { Maintain } \\
\text { Consuer / } \\
\text { personal Loan } \\
\text { Accounts }\end{array}$ & Branches & $\begin{array}{l}\text { Multi } \\
\text { User }\end{array}$ & $\begin{array}{l}\text { In } \\
\text { House }\end{array}$ & FoxPro 2.6 & $\begin{array}{l}\text { FoxPro } \\
2.6\end{array}$ & DOS & $\begin{array}{l}\text { Stand } \\
\text { Alone }\end{array}$ & Live \\
\hline & $\begin{array}{l}\text { Special saving } \\
\text { accounts } \\
\text { Application }\end{array}$ & $\begin{array}{l}\text { Maintain } \\
\text { Deposit } \\
\text { Schemes }\end{array}$ & Branches & $\begin{array}{l}\text { Multi } \\
\text { User }\end{array}$ & $\begin{array}{l}\text { In } \\
\text { House }\end{array}$ & FoxPro 2.6 & $\begin{array}{l}\text { FoxPro } \\
2.6\end{array}$ & DOS & $\begin{array}{l}\text { Stand } \\
\text { Alone }\end{array}$ & Live \\
\hline & Protection Shield & $\begin{array}{l}\text { Maintain } \\
\text { Deposit } \\
\text { Scheme }\end{array}$ & Branches & $\begin{array}{l}\text { Multi } \\
\text { User }\end{array}$ & $\begin{array}{l}\text { In } \\
\text { House }\end{array}$ & FoxPro 2.6 & $\begin{array}{l}\text { FoxPro } \\
2.6\end{array}$ & DOS & $\begin{array}{l}\text { Stand } \\
\text { Alone }\end{array}$ & Live \\
\hline & $\begin{array}{l}\text { Inter } \quad \text { Branch } \\
\text { account }\end{array}$ & $\begin{array}{l}\text { Data Entry } \\
\text { Software to } \\
\text { Prepare inter } \\
\text { branch } \\
\text { Account } \\
\text { Statement }\end{array}$ & Data Centers & $\begin{array}{l}\text { Multi } \\
\text { User }\end{array}$ & $\begin{array}{l}\text { In } \\
\text { House }\end{array}$ & FoxPro 2.6 & $\begin{array}{l}\text { FoxPro } \\
2.6\end{array}$ & DOS & $\begin{array}{l}\text { Stand } \\
\text { Alone }\end{array}$ & Live \\
\hline & $\begin{array}{l}\text { Basic } \quad \text { Bank } \\
\text { Accounts }\end{array}$ & $\begin{array}{l}\text { Deposit } \\
\text { Module } \\
\text { maintaining } \\
\text { Basic Bank } \\
\text { Account } \\
\text { Customers }\end{array}$ & Branches & $\begin{array}{l}\text { Multi } \\
\text { User }\end{array}$ & $\begin{array}{l}\text { In } \\
\text { House }\end{array}$ & FoxPro 2.6 & $\begin{array}{l}\text { FoxPro } \\
2.6\end{array}$ & DOS & $\begin{array}{l}\text { Stand } \\
\text { Alone }\end{array}$ & Live \\
\hline & $\begin{array}{l}\text { Inter Branch } \\
\text { Transactions }\end{array}$ & $\begin{array}{l}\text { Facilitate the } \\
\text { Inter Branch } \\
\text { Transaction at } \\
\text { all Online } \\
\text { branches }\end{array}$ & Branches & $\begin{array}{l}\text { Multi } \\
\text { User }\end{array}$ & $\begin{array}{l}\text { In } \\
\text { House }\end{array}$ & FoxPro 2.6 & $\begin{array}{l}\text { FoxPro } \\
2.6\end{array}$ & DOS & $\begin{array}{l}\text { Stand } \\
\text { Alone }\end{array}$ & Live \\
\hline & $\begin{array}{lr}\text { Inter } & \text { Branch } \\
\text { entries } & \text { in } \\
\text { Electronic } & \text { Banking } \\
\text { System (EBS) }\end{array}$ & $\begin{array}{l}\text { Developed } \\
\text { New Module } \\
\text { for auto Inter } \\
\text { Branch entries } \\
\text { of all EBS/ } \\
\text { Online } \\
\text { Branches }\end{array}$ & Branches & $\begin{array}{l}\text { Multi } \\
\text { User }\end{array}$ & $\begin{array}{l}\text { In } \\
\text { House }\end{array}$ & FoxPro 2.6 & $\begin{array}{l}\text { FoxPro } \\
2.6\end{array}$ & DOS & $\begin{array}{l}\text { Stand } \\
\text { Alone }\end{array}$ & Live \\
\hline & Withholding Tax & $\begin{array}{l}\text { Auto } \\
\text { Deduction of } \\
\text { Withholding } \\
\text { Tax from } \\
\text { customer } \\
\text { transactions }\end{array}$ & Branches & $\begin{array}{l}\text { Multi } \\
\text { User }\end{array}$ & $\begin{array}{l}\text { In } \\
\text { House }\end{array}$ & FoxPro 2.6 & $\begin{array}{l}\text { FoxPro } \\
2.6\end{array}$ & DOS & $\begin{array}{l}\text { Stand } \\
\text { Alone }\end{array}$ & Live \\
\hline & $\begin{array}{l}\text { Special saving } \\
\text { accounts } \\
\text { Application }\end{array}$ & $\begin{array}{l}\text { Maintainance } \\
\text { of Deposite } \\
\text { schemes }\end{array}$ & Data Centers & $\begin{array}{l}\text { Multi } \\
\text { User }\end{array}$ & $\begin{array}{l}\text { In } \\
\text { House }\end{array}$ & FoxPro 2.6 & $\begin{array}{l}\text { FoxPro } \\
2.6\end{array}$ & DOS & $\begin{array}{l}\text { Stand } \\
\text { Alone }\end{array}$ & Live \\
\hline & $\begin{array}{l}\text { Tax Collection } \\
\text { Automation } \\
\text { Software }\end{array}$ & $\begin{array}{l}\text { Handle All Tax } \\
\text { Payers Records }\end{array}$ & Branches & $\begin{array}{l}\text { Multi } \\
\text { User }\end{array}$ & $\begin{array}{l}\text { In } \\
\text { House }\end{array}$ & FoxPro 2.6 & $\begin{array}{l}\text { FoxPro } \\
2.6\end{array}$ & DOS & $\begin{array}{l}\text { Stand } \\
\text { Alone }\end{array}$ & Live \\
\hline & $\begin{array}{l}\text { Tax Collection } \\
\text { Automation } \\
\text { Software } \\
\text { forregulators }\end{array}$ & $\begin{array}{l}\text { Software to } \\
\text { handle all tax } \\
\text { payers for } \\
\text { State Bank of } \\
\text { Pakistan }\end{array}$ & $\begin{array}{l}\text { State Bank of } \\
\text { Pakistan }\end{array}$ & $\begin{array}{l}\text { Multi } \\
\text { User }\end{array}$ & $\begin{array}{l}\text { In } \\
\text { House }\end{array}$ & FoxPro 2.6 & $\begin{array}{l}\text { FoxPro } \\
2.6\end{array}$ & DOS & $\begin{array}{l}\text { Stand } \\
\text { Alone }\end{array}$ & Live \\
\hline & $\begin{array}{l}\text { Customer } \\
\text { Information } \\
\text { software }\end{array}$ & $\begin{array}{l}\text { Customer } \\
\text { Information } \\
\text { Containing } \\
\text { Software }\end{array}$ & Branches & $\begin{array}{l}\text { Multi } \\
\text { User }\end{array}$ & $\begin{array}{l}\text { In } \\
\text { House }\end{array}$ & FoxPro 2.6 & $\begin{array}{l}\text { FoxPro } \\
2.6\end{array}$ & DOS & $\begin{array}{l}\text { Stand } \\
\text { Alone }\end{array}$ & Live \\
\hline
\end{tabular}




\begin{tabular}{|c|c|c|c|c|c|c|c|c|c|c|}
\hline & $\begin{array}{l}\text { Govt. duties on } \\
\text { Banking Services } \\
\text { application }\end{array}$ & $\begin{array}{l}\text { All Govt. levies } \\
\text { management }\end{array}$ & Branches & $\begin{array}{l}\text { Multi } \\
\text { User }\end{array}$ & $\begin{array}{l}\text { In } \\
\text { House }\end{array}$ & FoxPro 2.6 & $\begin{array}{l}\text { FoxPro } \\
2.6\end{array}$ & DOS & $\begin{array}{l}\text { Stand } \\
\text { Alone }\end{array}$ & Live \\
\hline 3 & $\begin{array}{l}\text { Housing Loan } \\
\text { monitoring } \\
\text { application }\end{array}$ & $\begin{array}{l}\text { Consumer } \\
\text { Finance Loan } \\
\text { for Housing }\end{array}$ & Centralized & $\begin{array}{l}\text { Multi } \\
\text { user }\end{array}$ & $\begin{array}{l}\text { Out } \\
\text { Sourc } \\
\text { ed }\end{array}$ & $10 \mathrm{~g}$ suite & $\begin{array}{l}\text { Oracle10 } \\
\mathrm{g}\end{array}$ & DOS & On Line & Live \\
\hline 4 & $\begin{array}{l}\text { Retails Products } \\
\text { monitoring } \\
\text { application }\end{array}$ & $\begin{array}{l}\text { Consumer } \\
\text { Finance } \\
\text { software } \\
\text { covers retail } \\
\text { products } \\
\text { schemes }\end{array}$ & Centralized & $\begin{array}{l}\text { Multi } \\
\text { user }\end{array}$ & $\begin{array}{l}\text { Out } \\
\text { Sourc } \\
\text { ed }\end{array}$ & $10 \mathrm{~g}$ suite & $\begin{array}{l}\text { Oracle10 } \\
\mathrm{g}\end{array}$ & DOS & On Line & Live \\
\hline 5 & $\begin{array}{l}\text { Special Deposit } \\
\text { certificates }\end{array}$ & $\begin{array}{l}\text { Maintain } \\
\text { Deposit } \\
\text { Scheme }\end{array}$ & Centralized & $\begin{array}{l}\text { Multi } \\
\text { user }\end{array}$ & $\begin{array}{l}\text { In } \\
\text { House }\end{array}$ & $\begin{array}{ll}\text { ASP } & 3.0, \\
\text { ASP.Net, } & \\
\text { C\#.Net, } & \text { SSIS, } \\
\text { SSRS } & \end{array}$ & $\begin{array}{l}\text { SQL } \\
\text { Server } \\
2005\end{array}$ & Windows & On Line & Live \\
\hline 6 & $\begin{array}{l}\text { Online signature } \\
\text { System-I }\end{array}$ & $\begin{array}{l}\text { Verification of } \\
\text { customers } \\
\text { signatures }\end{array}$ & $\begin{array}{l}\text { Decentralize } \\
\mathrm{d}\end{array}$ & $\begin{array}{l}\text { Single } \\
\text { User }\end{array}$ & $\begin{array}{l}\text { In } \\
\text { House }\end{array}$ & VB 6.0 & $\begin{array}{l}\text { SQL } \\
\text { Server } \\
2000\end{array}$ & Windows & $\begin{array}{l}\text { Stand } \\
\text { Alone }\end{array}$ & Live \\
\hline 7 & $\begin{array}{l}\text { Online signature } \\
\text { System-II }\end{array}$ & $\begin{array}{l}\text { Signature } \\
\text { Verification of } \\
\text { all Online } \\
\text { branches to } \\
\text { handle Inter } \\
\text { Branch } \\
\text { Transactions }\end{array}$ & Centralized & & $\begin{array}{l}\text { In } \\
\text { House }\end{array}$ & VB 6.0 & $\begin{array}{l}\text { SQL } \\
\text { Server } \\
2000\end{array}$ & Windows & $\begin{array}{l}\text { Stand } \\
\text { Alone }\end{array}$ & Live \\
\hline 8 & $\begin{array}{l}\text { End of Day } \\
\text { Application }\end{array}$ & $\begin{array}{l}\text { Interface } \\
\text { between Retail } \\
\text { and deposit } \\
\text { and other web } \\
\text { based deposit } \\
\text { schemes, } \\
\text { posting of } \\
\text { financial } \\
\text { transaction of } \\
\text { different } \\
\text { products }\end{array}$ & Centralized & $\begin{array}{l}\text { Multi } \\
\text { User }\end{array}$ & $\begin{array}{l}\text { In } \\
\text { House }\end{array}$ & ASP.Net,C\# & $\begin{array}{l}\text { Oracle10 } \\
\mathrm{g}\end{array}$ & Windows & & Live \\
\hline 9 & $\begin{array}{l}\text { Retail Product } \\
\text { monitoring } \\
\text { software }\end{array}$ & $\begin{array}{l}\text { Revamped } \\
\text { Retail } \\
\text { Application to } \\
\text { be used in } \\
\text { Branch Back } \\
\text { Office }\end{array}$ & $\begin{array}{l}\text { Decentralize } \\
\mathrm{d}\end{array}$ & $\begin{array}{l}\text { Multi } \\
\text { User }\end{array}$ & $\begin{array}{l}\text { In } \\
\text { House }\end{array}$ & ASP.Net,C\# & $\begin{array}{l}\text { Oracle10 } \\
\mathrm{g}\end{array}$ & Windows & & Live \\
\hline 10 & $\begin{array}{l}\text { Financial } \\
\text { statements System }\end{array}$ & $\begin{array}{l}\text { System } \\
\text { Generated } \\
\text { documents, } \\
\text { Synchronized } \\
\text { Document } \\
\text { template } \\
\text { across the } \\
\text { Country, } \\
\text { Removal of } \\
\text { dependency on } \\
\text { Bank Legal } \\
\text { Officers }\end{array}$ & Centralized & $\begin{array}{l}\text { Multi } \\
\text { user }\end{array}$ & $\begin{array}{l}\text { In } \\
\text { House }\end{array}$ & ASP.Net, C\# & $\begin{array}{l}\text { SQL } \\
\text { Server } \\
2005\end{array}$ & Windows & On Line & $\begin{array}{l}\text { un } \\
\text { operat } \\
\text { ional }\end{array}$ \\
\hline 11 & $\begin{array}{l}\text { Pledged Stock } \\
\text { Management } \\
\text { System }\end{array}$ & $\begin{array}{l}\text { Computerizati } \\
\text { on of Seasonal, } \\
\text { running, Cash } \\
\text { Finances and } \\
\text { to monitor the } \\
\text { stock } \\
\text { shortages and } \\
\text { Shortfalls }\end{array}$ & Centralized & $\begin{array}{l}\text { Multi } \\
\text { user }\end{array}$ & $\begin{array}{l}\text { In } \\
\text { House }\end{array}$ & ASP.Net, C\# & $\begin{array}{l}\text { SQL } \\
\text { Server } \\
2005\end{array}$ & Windows & On Line & $\begin{array}{l}\text { Under } \\
\text { Develo } \\
\text { pment }\end{array}$ \\
\hline 12 & Stock for stationary & $\begin{array}{l}\text { Stationary } \\
\text { Inventory } \\
\text { Positions and } \\
\text { Reports }\end{array}$ & Centralized & $\begin{array}{l}\text { Multi } \\
\text { user }\end{array}$ & $\begin{array}{l}\text { In } \\
\text { House }\end{array}$ & ASP.Net, C\# & $\begin{array}{l}\text { SQL } \\
\text { Server } \\
2005\end{array}$ & Windows & On Line & Live \\
\hline 13 & $\begin{array}{ll}\text { Office } & \text { Files } \\
\text { Management } & \\
\text { System } & \end{array}$ & $\begin{array}{l}\text { To automate } \\
\text { the office file } \\
\text { system and } \\
\text { their contents }\end{array}$ & Centralized & $\begin{array}{l}\text { Multi } \\
\text { User }\end{array}$ & $\begin{array}{l}\text { In } \\
\text { House }\end{array}$ & ASP.Net, C\# & $\begin{array}{l}\text { SQL } \\
\text { Server } \\
2006\end{array}$ & Windows & On Line & $\begin{array}{l}\text { un } \\
\text { operat } \\
\text { ional }\end{array}$ \\
\hline 14 & Benevolent Fund & $\begin{array}{l}\text { Computerizati } \\
\text { on and } \\
\text { centralization } \\
\text { of all } \\
\text { Benevolent } \\
\text { fund } \\
\text { accumulation }\end{array}$ & Centralized & $\begin{array}{l}\text { Multi } \\
\text { User }\end{array}$ & $\begin{array}{l}\text { In } \\
\text { House }\end{array}$ & ASP.Net, C\# & $\begin{array}{l}\text { SQL } \\
\text { Server } \\
2007\end{array}$ & Windows & On Line & $\begin{array}{l}\text { Under } \\
\text { Develo } \\
\text { pment }\end{array}$ \\
\hline
\end{tabular}




\begin{tabular}{|c|c|c|c|c|c|c|c|c|c|c|}
\hline 15 & $\begin{array}{l}\text { Transactions } \\
\text { Merging Software }\end{array}$ & $\begin{array}{l}\text { Compilation of } \\
\text { Off-line } \\
\text { branches } \\
\text { transactions }\end{array}$ & $\begin{array}{lr}\text { Data } & \text { Centers } \\
\text { and } & \text { Head } \\
\text { Office } & \end{array}$ & $\begin{array}{l}\text { Single } \\
\text { User }\end{array}$ & $\begin{array}{l}\text { In } \\
\text { House }\end{array}$ & FoxPro 2.6 & $\begin{array}{l}\text { FoxPro } \\
2.6\end{array}$ & DOS & $\begin{array}{l}\text { Stand } \\
\text { Alone }\end{array}$ & Live \\
\hline 16 & $\begin{array}{l}\text { Statement of } \\
\text { Financial Affairs \& } \\
\text { profit and losses }\end{array}$ & $\begin{array}{l}\text { Prepare } \\
\text { Branch Wise } \\
\text { Profit \& Loss/ } \\
\text { Statement of } \\
\text { Affairs }\end{array}$ & $\begin{array}{l}\text { Decentralize } \\
\mathrm{d}\end{array}$ & $\begin{array}{l}\text { Single } \\
\text { User }\end{array}$ & $\begin{array}{l}\text { In } \\
\text { House }\end{array}$ & & & & $\begin{array}{l}\text { Stand } \\
\text { Alone }\end{array}$ & Live \\
\hline 17 & $\begin{array}{l}\text { Financial } \\
\text { statements System- } \\
\text { II }\end{array}$ & $\begin{array}{l}\text { Consolidation } \\
\text { of } \quad \text { Finance } \\
\text { related reports }\end{array}$ & $\begin{array}{l}\text { Branches, } \\
\text { Regional \& } \\
\text { Head office }\end{array}$ & $\begin{array}{l}\text { Single } \\
\text { User }\end{array}$ & $\begin{array}{l}\text { In } \\
\text { House }\end{array}$ & & & & $\begin{array}{l}\text { Stand } \\
\text { Alone }\end{array}$ & Live \\
\hline 18 & $\begin{array}{ll}\text { Long } & \text { Term } \\
\text { Finance } & \\
\text { Application } & \end{array}$ & $\begin{array}{l}\text { Maintain Staff } \\
\text { Advances }\end{array}$ & $\begin{array}{l}\text { Main Branch } \\
\text { Karachi }\end{array}$ & $\begin{array}{l}\text { Single } \\
\text { User }\end{array}$ & $\begin{array}{l}\text { In } \\
\text { House }\end{array}$ & & & & $\begin{array}{l}\text { Stand } \\
\text { Alone }\end{array}$ & $\begin{array}{l}\text { un } \\
\text { operat } \\
\text { ional }\end{array}$ \\
\hline 19 & $\begin{array}{l}\text { Online } \quad \text { Funds } \\
\text { Transfer System }\end{array}$ & $\begin{array}{l}\text { Remittances of } \\
\text { Online } \\
\text { Branches }\end{array}$ & $\begin{array}{l}\text { Main Branch } \\
\text { Karachi }\end{array}$ & $\begin{array}{l}\text { Multi } \\
\text { user }\end{array}$ & $\begin{array}{l}\text { In } \\
\text { House }\end{array}$ & & & & $\begin{array}{l}\text { Stand } \\
\text { Alone }\end{array}$ & Live \\
\hline 20 & $\begin{array}{l}\text { Assets } \quad \text { and } \\
\text { Liablities } \\
\text { Management } \\
\text { System-I }\end{array}$ & $\begin{array}{lc}\text { Data Entry } & \text { of } \\
\text { Assets } & \& \\
\text { Liabilities } & \end{array}$ & $\begin{array}{l}\text { Branches, } \\
\text { Regional \& } \\
\text { Head office }\end{array}$ & $\begin{array}{l}\text { Multi } \\
\text { user }\end{array}$ & $\begin{array}{l}\text { In } \\
\text { House }\end{array}$ & & & & $\begin{array}{l}\text { Stand } \\
\text { Alone }\end{array}$ & \\
\hline 21 & $\begin{array}{l}\text { Classified } \\
\text { Accounts } \\
\text { monitoring System }\end{array}$ & $\begin{array}{l}\text { Data Entry for } \\
\text { Classified } \\
\text { Advances }\end{array}$ & $\begin{array}{l}\text { All Regional } \\
\text { Offices }\end{array}$ & $\begin{array}{l}\text { Multi } \\
\text { user }\end{array}$ & $\begin{array}{l}\text { In } \\
\text { House }\end{array}$ & & & & $\begin{array}{l}\text { Stand } \\
\text { Alone }\end{array}$ & \\
\hline 22 & $\begin{array}{l}\text { Weekly } \\
\text { information Charts }\end{array}$ & $\begin{array}{l}\text { Data Entry of } \\
\text { Assets } \\
\text { Liabilities for } \\
\text { onward } \\
\text { submission to } \\
\text { Regulators }\end{array}$ & $\begin{array}{l}\text { Branches, } \\
\text { Regional \& } \\
\text { Head office }\end{array}$ & $\begin{array}{l}\text { Multi } \\
\text { user }\end{array}$ & $\begin{array}{l}\text { In } \\
\text { House }\end{array}$ & & & & $\begin{array}{l}\text { Stand } \\
\text { Alone }\end{array}$ & \\
\hline 23 & $\begin{array}{l}\text { Regulator's data } \\
\text { for loan Accounts } \\
\text { (ECIB) }\end{array}$ & $\begin{array}{l}\text { Data capturing } \\
\text { for Advances } \\
\text { Customer's for } \\
\text { Regulators }\end{array}$ & $\begin{array}{l}\text { Branches, } \\
\text { Regional \& } \\
\text { Head office }\end{array}$ & $\begin{array}{l}\text { Single } \\
\text { User }\end{array}$ & $\begin{array}{l}\text { In } \\
\text { House }\end{array}$ & & & & $\begin{array}{l}\text { Stand } \\
\text { Alone }\end{array}$ & \\
\hline 24 & $\begin{array}{l}\text { Foreign } \\
\text { remittances } \\
\text { Reconciliation } \\
\text { application }\end{array}$ & $\begin{array}{l}\text { Maintain } \\
\text { Foreign } \\
\text { transactions } \\
\text { Reconciliation }\end{array}$ & $\begin{array}{l}\text { Operation } \\
\text { Group }\end{array}$ & $\begin{array}{l}\text { Single } \\
\text { User }\end{array}$ & $\begin{array}{l}\text { In } \\
\text { House }\end{array}$ & Visual FoxPro & & Windows & $\begin{array}{l}\text { Stand } \\
\text { Alone }\end{array}$ & Live \\
\hline 25 & $\begin{array}{l}\text { Foreign Exchange } \\
\text { Vouching } \\
\text { Application }\end{array}$ & $\begin{array}{l}\text { Data Entry of } \\
\text { Foreign } \\
\text { Exchange } \\
\text { Vouchers }\end{array}$ & Main Branch & $\begin{array}{l}\text { Single } \\
\text { User }\end{array}$ & $\begin{array}{l}\text { In } \\
\text { House }\end{array}$ & FoxPro & & DOS & $\begin{array}{l}\text { Stand } \\
\text { Alone }\end{array}$ & Live \\
\hline 26 & $\begin{array}{l}\text { Foreign } \\
\text { Remittance } \\
\text { management } \\
\text { System }\end{array}$ & $\begin{array}{l}\text { Maintain } \\
\text { Inward } \\
\text { Foreign } \\
\text { Remittances } \\
\text { Records }\end{array}$ & Main Branch & $\begin{array}{l}\text { Single } \\
\text { User }\end{array}$ & $\begin{array}{l}\text { In } \\
\text { House }\end{array}$ & & & & $\begin{array}{l}\text { Stand } \\
\text { Alone }\end{array}$ & \\
\hline 27 & $\begin{array}{l}\text { Inland } \quad \text { Bills } \\
\text { management } \\
\text { System }\end{array}$ & $\begin{array}{l}\text { Maintain Bills } \\
\text { Data Entries } \\
\text { and } \\
\text { Consolidation }\end{array}$ & Main Branch & $\begin{array}{l}\text { Single } \\
\text { User }\end{array}$ & $\begin{array}{l}\text { In } \\
\text { House }\end{array}$ & VB.Net & $\begin{array}{l}\text { SQL } \\
\text { Server }\end{array}$ & Windows & $\begin{array}{l}\text { Stand } \\
\text { Alone }\end{array}$ & Live \\
\hline 28 & $\begin{array}{l}\text { traveller Cheques } \\
\text { monitoring system }\end{array}$ & $\begin{array}{l}\text { Maintain } \\
\text { Traveller } \\
\text { Cheques } \\
\text { records }\end{array}$ & Branch & $\begin{array}{l}\text { Single } \\
\text { User }\end{array}$ & $\begin{array}{l}\text { In } \\
\text { House }\end{array}$ & FoxPro (Dos) & & DOS & $\begin{array}{l}\text { Stand } \\
\text { Alone }\end{array}$ & Live \\
\hline 29 & $\begin{array}{ll}\text { Tax } & \text { Collection } \\
\text { System } & \end{array}$ & $\begin{array}{l}\text { Tax Collection } \\
\text { Data } \\
\text { Compilation } \\
\text { for transfer }\end{array}$ & Head Office & $\begin{array}{l}\text { Multi } \\
\text { user }\end{array}$ & $\begin{array}{l}\text { Out } \\
\text { Sourc } \\
\text { ed }\end{array}$ & & & & & Live \\
\hline 30 & $\begin{array}{l}\text { Large Tax Payers } \\
\text { management } \\
\text { System-I }\end{array}$ & $\begin{array}{l}\text { Handle Large } \\
\text { Tax Unit (Tax } \\
\text { Payers of Sales } \\
\text { Tax through } \\
\text { Web) }\end{array}$ & Branches & $\begin{array}{l}\text { Multi } \\
\text { user }\end{array}$ & $\begin{array}{l}\text { Out } \\
\text { Sourc } \\
\text { ed }\end{array}$ & & & & & Live \\
\hline 31 & $\begin{array}{l}\text { Large Tax Payers } \\
\text { management } \\
\text { System-II }\end{array}$ & $\begin{array}{l}\text { Large Tax Unit } \\
\text { (Web base } \\
\text { application to } \\
\text { handle Sales } \\
\text { Tax Returns) }\end{array}$ & $\begin{array}{l}\text { Main } \\
\text { Branches of } \\
\text { Karachi \& } \\
\text { Lahore }\end{array}$ & $\begin{array}{l}\text { Multi } \\
\text { user }\end{array}$ & $\begin{array}{l}\text { Out } \\
\text { Sourc } \\
\text { ed }\end{array}$ & & & & & Live \\
\hline 32 & $\begin{array}{l}\text { Human Resource } \\
\text { Mangement } \\
\text { System }\end{array}$ & $\begin{array}{l}\text { Maintain } \\
\text { Human } \\
\text { Resources Data }\end{array}$ & HRM Group & $\begin{array}{l}\text { Single } \\
\text { User }\end{array}$ & $\begin{array}{l}\text { In } \\
\text { House }\end{array}$ & & & & & Live \\
\hline 33 & $\begin{array}{l}\text { HR Medical } \\
\text { Facility } \\
\text { Management }\end{array}$ & $\begin{array}{l}\text { Maintain Staff } \\
\text { Medical } \\
\text { Records }\end{array}$ & HRM Group & $\begin{array}{l}\text { Single } \\
\text { User }\end{array}$ & $\begin{array}{l}\text { In } \\
\text { House }\end{array}$ & & & & & $\begin{array}{l}\text { Under } \\
\text { Develo } \\
\text { pment }\end{array}$ \\
\hline
\end{tabular}


System

\begin{tabular}{|c|c|c|c|c|c|c|c|c|c|c|}
\hline 34 & $\begin{array}{l}\text { Retired Employees } \\
\text { Facilities System }\end{array}$ & $\begin{array}{l}\text { Maintain } \\
\text { retired } \\
\text { Records }\end{array}$ & Head Office & $\begin{array}{l}\text { Single } \\
\text { User }\end{array}$ & $\begin{array}{l}\text { In } \\
\text { House }\end{array}$ & & & & & $\begin{array}{l}\text { Under } \\
\text { Develo } \\
\text { pment }\end{array}$ \\
\hline 35 & $\begin{array}{l}\text { Banks' standard } \\
\text { Stationary System }\end{array}$ & $\begin{array}{l}\text { Data Entry of } \\
\text { Banks' } \\
\text { Standard } \\
\text { Documents }\end{array}$ & $\begin{array}{l}\text { Operation } \\
\text { Group }\end{array}$ & $\begin{array}{l}\text { Single } \\
\text { User }\end{array}$ & $\begin{array}{l}\text { In } \\
\text { House }\end{array}$ & Visual FoxPro & & Windows & & $\begin{array}{l}\text { Under } \\
\text { Develo } \\
\text { pment }\end{array}$ \\
\hline 36 & $\begin{array}{l}\text { Banks' Vehicles } \\
\text { Management } \\
\text { System }\end{array}$ & $\begin{array}{l}\text { Maintain Bank } \\
\text { Vehicle record }\end{array}$ & $\begin{array}{l}\text { Operation } \\
\text { Group }\end{array}$ & $\begin{array}{l}\text { Single } \\
\text { User }\end{array}$ & $\begin{array}{l}\text { In } \\
\text { House }\end{array}$ & Visual FoxPro & & Windows & & Live \\
\hline 37 & Balloting System & $\begin{array}{l}\text { Maintains } \\
\text { Different } \\
\text { Balloting } \\
\text { Results }\end{array}$ & $\begin{array}{l}\text { Operation } \\
\text { Group }\end{array}$ & $\begin{array}{l}\text { Multi } \\
\text { user }\end{array}$ & $\begin{array}{l}\text { In } \\
\text { House }\end{array}$ & FoxPro (DOS) & & DOS & $\begin{array}{l}\text { Stand } \\
\text { Alone }\end{array}$ & Live \\
\hline 38 & $\begin{array}{l}\text { Dividend Payment } \\
\text { Software }\end{array}$ & $\begin{array}{l}\text { Handle data } \\
\text { entry and } \\
\text { reconciliation } \\
\text { of different } \\
\text { companies } \\
\text { dividend } \\
\text { vouchers }\end{array}$ & Branches & $\begin{array}{l}\text { Multi } \\
\text { user }\end{array}$ & $\begin{array}{l}\text { In } \\
\text { House }\end{array}$ & VB 6.0 & $\begin{array}{l}\text { SQL } \\
\text { Server } \\
2000\end{array}$ & Windows & $\begin{array}{l}\text { Stand } \\
\text { Alone }\end{array}$ & Live \\
\hline 39 & $\begin{array}{l}\text { MIS Reports } \\
\text { Application }\end{array}$ & $\begin{array}{l}\text { Consolidation } \\
\text { of all branches } \\
\text { data received } \\
\text { from data } \\
\text { centers and } \\
\text { generation of } \\
\text { reports }\end{array}$ & $\begin{array}{l}\text { Financial } \\
\text { Control } \\
\text { Group }\end{array}$ & $\begin{array}{l}\text { Multi } \\
\text { user }\end{array}$ & $\begin{array}{l}\text { In } \\
\text { House }\end{array}$ & VB 6.0 & $\begin{array}{l}\text { SQL } \\
\text { Server } \\
2000\end{array}$ & Windows & $\begin{array}{l}\text { Stand } \\
\text { Alone }\end{array}$ & Live \\
\hline 40 & $\begin{array}{l}\text { Fixed Assets } \\
\text { Management } \\
\text { System }\end{array}$ & $\begin{array}{l}\text { Auto } \\
\text { calculation of } \\
\text { assets book } \\
\text { value \& } \\
\text { generates } \\
\text { Depreciation. }\end{array}$ & $\begin{array}{l}\text { Financial } \\
\text { Control } \\
\text { Group }\end{array}$ & $\begin{array}{l}\text { Multi } \\
\text { user }\end{array}$ & $\begin{array}{l}\text { In } \\
\text { House }\end{array}$ & ASP.Net,VB.Net & $\begin{array}{l}\text { SQL } \\
\text { Server } \\
2005\end{array}$ & Windows & On Line & Live \\
\hline 41 & $\begin{array}{l}\text { Branch Operations } \\
\text { Log Generation } \\
\text { System }\end{array}$ & $\begin{array}{l}\text { Information } \\
\text { received from } \\
\text { all Data } \\
\text { centers used to } \\
\text { generate } \\
\text { reports } \\
\text { showing } \\
\text { modules being } \\
\text { used by the } \\
\text { branches }\end{array}$ & Head Office & $\begin{array}{l}\text { Multi } \\
\text { user }\end{array}$ & $\begin{array}{l}\text { In } \\
\text { House }\end{array}$ & VB.Net & $\begin{array}{l}\text { SQL } \\
\text { Server }\end{array}$ & Windows & On Line & Live \\
\hline 42 & $\begin{array}{l}\text { Direct Debit } \\
\text { Facility } \\
\text { Management } \\
\text { System }\end{array}$ & $\begin{array}{l}\text { Direct Debit } \\
\text { Facility } \\
\text { customers }\end{array}$ & Branches & $\begin{array}{l}\text { Multi } \\
\text { user }\end{array}$ & $\begin{array}{l}\text { In } \\
\text { House }\end{array}$ & VB.Net & $\begin{array}{l}\text { SQL } \\
\text { Server }\end{array}$ & Windows & On Line & Live \\
\hline 43 & $\begin{array}{l}\text { Utility } \\
\text { Payment }\end{array}$ & $\begin{array}{lr}\text { Utility } & \text { Bills } \\
\text { Payment } & \\
\text { receipt } & \text { data } \\
\text { entry } & \text { and } \\
\text { transfer } & \end{array}$ & Branches & $\begin{array}{l}\text { Multi } \\
\text { user }\end{array}$ & $\begin{array}{l}\text { Out } \\
\text { Sourc } \\
\text { ed }\end{array}$ & & & & $\begin{array}{l}\text { Online } \\
\text { (internet) }\end{array}$ & Live \\
\hline 44 & $\begin{array}{l}\text { Local Tax Payment } \\
\text { System }\end{array}$ & $\begin{array}{l}\text { Maintains local } \\
\text { tax payment } \\
\text { records }\end{array}$ & Branches & $\begin{array}{l}\text { Multi } \\
\text { user }\end{array}$ & $\begin{array}{l}\text { In } \\
\text { House }\end{array}$ & & & & $\begin{array}{l}\text { Stand } \\
\text { Alone }\end{array}$ & $\begin{array}{l}\text { un } \\
\text { operat } \\
\text { ional }\end{array}$ \\
\hline 45 & $\begin{array}{l}\text { Treasury } \\
\text { Management } \\
\text { System }\end{array}$ & $\begin{array}{l}\text { Treasury } \\
\text { management }\end{array}$ & $\begin{array}{l}\text { Treasury } \\
\text { Group }\end{array}$ & $\begin{array}{l}\text { Multi } \\
\text { user }\end{array}$ & $\begin{array}{l}\text { In } \\
\text { House }\end{array}$ & & & & $\begin{array}{l}\text { Stand } \\
\text { Alone }\end{array}$ & Live \\
\hline 46 & $\begin{array}{l}\text { Inventory } \\
\text { Standard } \\
\text { Documents }\end{array}$ & $\begin{array}{l}\text { Maintains } \\
\text { inventory of } \\
\text { Banks' } \\
\text { Standard } \\
\text { documents }\end{array}$ & Head Office & $\begin{array}{l}\text { Single } \\
\text { User }\end{array}$ & $\begin{array}{l}\text { In } \\
\text { House }\end{array}$ & & & & $\begin{array}{l}\text { Stand } \\
\text { Alone }\end{array}$ & Live \\
\hline 47 & $\begin{array}{l}\text { Activity Recording } \\
\text { System for Central } \\
\text { Dispatch Dep't }\end{array}$ & $\begin{array}{l}\text { Data } \\
\text { management\& } \\
\text { processing for } \\
\text { Logistics } \\
\text { Central } \\
\text { Dispatch Dept. }\end{array}$ & $\begin{array}{l}\text { Decentralize } \\
\mathrm{d}\end{array}$ & $\begin{array}{l}\text { Single } \\
\text { User }\end{array}$ & $\begin{array}{l}\text { In } \\
\text { House }\end{array}$ & C\#.Net & $\begin{array}{l}\text { SQL } \\
\text { Server } \\
2000\end{array}$ & Windows & $\begin{array}{l}\text { Stand } \\
\text { Alone }\end{array}$ & Live \\
\hline 48 & $\begin{array}{l}\text { Credit } \\
\text { Administration } \\
\text { and Monitoring } \\
\text { System }\end{array}$ & $\begin{array}{l}\text { Maintains } \\
\text { operational } \\
\text { information } \\
\text { and activities }\end{array}$ & CAD Group & $\begin{array}{l}\text { Single } \\
\text { User }\end{array}$ & $\begin{array}{l}\text { In } \\
\text { House }\end{array}$ & & & & $\begin{array}{l}\text { Stand } \\
\text { Alone }\end{array}$ & $\begin{array}{l}\text { un } \\
\text { operat } \\
\text { ional }\end{array}$ \\
\hline
\end{tabular}




\begin{tabular}{|c|c|c|c|c|c|c|c|c|c|c|}
\hline 49 & $\begin{array}{l}\text { Collateral Data } \\
\text { management-I }\end{array}$ & $\begin{array}{l}\text { Maintains } \\
\text { Collateral } \\
\text { Information }\end{array}$ & CAD Group & $\begin{array}{l}\text { Single } \\
\text { User }\end{array}$ & $\begin{array}{l}\text { In } \\
\text { House }\end{array}$ & & & & $\begin{array}{l}\text { Stand } \\
\text { Alone }\end{array}$ & $\begin{array}{l}\text { un } \\
\text { operat } \\
\text { ional }\end{array}$ \\
\hline 50 & $\begin{array}{l}\text { Government } \\
\text { Transaction } \\
\text { Account }\end{array}$ & $\begin{array}{l}\text { Direct } \\
\text { Transactions } \\
\text { with } \\
\text { Government }\end{array}$ & $\begin{array}{l}\text { Main } \\
\text { Branches }\end{array}$ & $\begin{array}{l}\text { Single } \\
\text { User }\end{array}$ & $\begin{array}{l}\text { In } \\
\text { House }\end{array}$ & & & & $\begin{array}{l}\text { Stand } \\
\text { Alone }\end{array}$ & Live \\
\hline 51 & $\begin{array}{l}\text { Govt. Accounts } \\
\text { System }\end{array}$ & $\begin{array}{l}\text { Direct } \\
\text { Transactions } \\
\text { with Govt. }\end{array}$ & $\begin{array}{l}\text { Main } \\
\text { Branches }\end{array}$ & $\begin{array}{l}\text { Single } \\
\text { User }\end{array}$ & $\begin{array}{l}\text { In } \\
\text { House }\end{array}$ & Fox Pro & Fox Pro & Windows & $\begin{array}{l}\text { Stand } \\
\text { Alone }\end{array}$ & Live \\
\hline 52 & $\begin{array}{l}\text { Government } \\
\text { Instruments } \\
\text { Management } \\
\text { System }\end{array}$ & $\begin{array}{l}\text { Payment and } \\
\text { collection of } \\
\text { Government } \\
\text { instruments }\end{array}$ & Head Office & $\begin{array}{l}\text { Single } \\
\text { User }\end{array}$ & $\begin{array}{l}\text { In } \\
\text { House }\end{array}$ & Fox Pro & Fox Pro & Windows & $\begin{array}{l}\text { Stand } \\
\text { Alone }\end{array}$ & $\begin{array}{l}\text { un } \\
\text { operat } \\
\text { ional }\end{array}$ \\
\hline 53 & $\begin{array}{l}\text { Audit Management } \\
\text { System }\end{array}$ & $\begin{array}{l}\text { Maintains } \\
\text { record of audit } \\
\text { irregularities } \\
\text { and } \\
\text { rectification } \\
\text { status }\end{array}$ & Audit Group & $\begin{array}{l}\text { Multi } \\
\text { User }\end{array}$ & $\begin{array}{l}\text { In } \\
\text { House }\end{array}$ & & & & $\begin{array}{l}\text { Stand } \\
\text { Alone }\end{array}$ & Live \\
\hline 54 & $\begin{array}{l}\text { Collateral Data } \\
\text { management-II }\end{array}$ & $\begin{array}{l}\text { Inventory of } \\
\text { Collateral } \\
\text { Property } \\
\text { Document }\end{array}$ & Centralized & $\begin{array}{l}\text { Multi } \\
\text { User }\end{array}$ & $\begin{array}{l}\text { In } \\
\text { House }\end{array}$ & ASP.Net, C\# & $\begin{array}{l}\text { SQL } \\
\text { Server }\end{array}$ & Windows & On Line & $\begin{array}{l}\text { Under } \\
\text { Develo } \\
\text { pment }\end{array}$ \\
\hline 55 & $\begin{array}{l}\text { Advances } \\
\text { Recovery \& } \\
\text { Default application }\end{array}$ & $\begin{array}{l}\text { It collect the } \\
\text { delinquent } \\
\text { data from } \\
\text { online / offline } \\
\text { system }\end{array}$ & Centralized & $\begin{array}{l}\text { Multi } \\
\text { User }\end{array}$ & $\begin{array}{l}\text { In } \\
\text { House }\end{array}$ & ASP.Net, C\# & $\begin{array}{l}\text { SQL } \\
\text { Server }\end{array}$ & Windows & On Line & Live \\
\hline 56 & $\begin{array}{l}\text { Quarterly } \\
\text { Statement } \\
\text { Affairs }\end{array}$ & $\begin{array}{l}\text { It generate } \\
\text { different kind } \\
\text { of reporting }\end{array}$ & $\begin{array}{l}\text { Decentralize } \\
\mathrm{d}\end{array}$ & $\begin{array}{l}\text { Multi } \\
\text { User }\end{array}$ & $\begin{array}{l}\text { In } \\
\text { House }\end{array}$ & ASP.Net, C\# & $\begin{array}{l}\text { SQL } \\
\text { Server }\end{array}$ & Windows & On Line & Live \\
\hline 57 & $\begin{array}{l}\text { Subsidiaries } \\
\text { Account }\end{array}$ & $\begin{array}{l}\text { Inventory of } \\
\text { Subsidiaries } \\
\text { Accounts }\end{array}$ & Centralized & $\begin{array}{l}\text { Multi } \\
\text { User }\end{array}$ & $\begin{array}{l}\text { In } \\
\text { House }\end{array}$ & ASP.Net, C\# & $\begin{array}{l}\text { SQL } \\
\text { Server }\end{array}$ & Windows & On Line & $\begin{array}{l}\text { Compl } \\
\text { eted } \\
\text { But On } \\
\text { Hold }\end{array}$ \\
\hline 58 & $\begin{array}{l}\text { Basel } \\
\text { Authorization } \\
\text { Management } \\
\text { System }\end{array}$ & $\begin{array}{l}\text { Basel } \\
\text { document } \\
\text { authorization } \\
\text { management } \\
\text { system }\end{array}$ & Centralized & $\begin{array}{l}\text { Multi } \\
\text { User }\end{array}$ & $\begin{array}{l}\text { In } \\
\text { House }\end{array}$ & ASP.Net, C\# & $\begin{array}{l}\text { SQL } \\
\text { Server }\end{array}$ & Windows & On Line & Live \\
\hline 59 & $\begin{array}{l}\text { E Banking Services } \\
\text { Management } \\
\text { System }\end{array}$ & $\begin{array}{l}\text { It is e-Banking } \\
\text { system which } \\
\text { facilitates the } \\
\text { account } \\
\text { holders though } \\
\text { web and } \\
\text { mobile. }\end{array}$ & Centralized & $\begin{array}{l}\text { Multi } \\
\text { User }\end{array}$ & $\begin{array}{l}\text { In } \\
\text { House }\end{array}$ & ASP.Net, C\# & $\begin{array}{l}\text { SQL } \\
\text { Server } \\
2005\end{array}$ & Windows & On Line & $\begin{array}{l}\text { On } \\
\text { Hold }\end{array}$ \\
\hline 60 & $\begin{array}{l}\text { General } \\
\text { Allowances }\end{array}$ & $\begin{array}{l}\text { Auto } \\
\text { calculation of } \\
\text { allowances, } \\
\text { Monthly } \\
\text { Reporting }\end{array}$ & Centralized & $\begin{array}{l}\text { Multi } \\
\text { User }\end{array}$ & $\begin{array}{l}\text { In } \\
\text { House }\end{array}$ & ASP.Net, C\# & $\begin{array}{l}\text { SQL } \\
\text { Server } \\
2006\end{array}$ & Windows & On Line & Live \\
\hline 61 & Provident Fund & $\begin{array}{l}\text { Provident } \\
\text { Fund collection } \\
\text { from employee } \\
\text { salary, accrual } \\
\text { of growth on } \\
\text { fund and } \\
\text { maintaining } \\
\text { record of loan } \\
\text { against PF }\end{array}$ & Centralized & $\begin{array}{l}\text { Multi } \\
\text { Users }\end{array}$ & $\begin{array}{l}\text { In- } \\
\text { house }\end{array}$ & COBOL AS400 & DBII/36 & $\begin{array}{l}\text { AS400/ } \\
\text { OS400 }\end{array}$ & $\begin{array}{l}\text { Stand } \\
\text { Alone }\end{array}$ & $\begin{array}{l}\text { Operat } \\
\text { ional }\end{array}$ \\
\hline 62 & Payroll & $\begin{array}{l}\text { Preparation of } \\
\text { Salary }\end{array}$ & $\begin{array}{l}\text { Decentralize } \\
\mathrm{d}\end{array}$ & Single & $\begin{array}{l}\text { In- } \\
\text { house }\end{array}$ & COBOL AS400 & $\begin{array}{l}\text { Index } \\
\text { Files }\end{array}$ & $\begin{array}{l}\text { AS400/ } \\
\text { OS400 }\end{array}$ & $\begin{array}{l}\text { Stand } \\
\text { Alone }\end{array}$ & $\begin{array}{l}\text { Operat } \\
\text { ional }\end{array}$ \\
\hline 63 & $\begin{array}{l}\text { EOBI Data } \\
\text { Management } \\
\text { System }\end{array}$ & $\begin{array}{l}\text { EOBI DATA } \\
\text { MANAGEMENT } \\
\text { SYSTEM }\end{array}$ & Centralized & Single & $\begin{array}{l}\text { In } \\
\text { House }\end{array}$ & $\mathrm{C \#}$ & Access & Windows & & $\begin{array}{l}\text { Deploy } \\
\text { ment } \\
\text { Phase }\end{array}$ \\
\hline 64 & $\begin{array}{l}\text { Web Based Basel II } \\
\text { Application (WBA) }\end{array}$ & $\begin{array}{l}\text { Data capturing } \\
\text { for Basel II }\end{array}$ & Centralized & $\begin{array}{l}\text { Multi } \\
\text { User }\end{array}$ & $\begin{array}{l}\text { Out } \\
\text { Sourc } \\
\text { ed }\end{array}$ & C\#, Asp.net & $\begin{array}{l}\text { SQL } \\
\text { Server } \\
2000\end{array}$ & Windows & On Line & Live \\
\hline 65 & $\begin{array}{l}\text { Annual Closing } \\
\text { Software }\end{array}$ & $\begin{array}{l}\text { Consolidation } \\
\text { of profit and } \\
\text { loss, statement } \\
\text { of affairs and } \\
\text { subsidiary } \\
\text { statements at } \\
\text { year end }\end{array}$ & $\begin{array}{l}\text { Decentralize } \\
\mathrm{d}\end{array}$ & $\begin{array}{l}\text { Single } \\
\text { User }\end{array}$ & $\begin{array}{l}\text { In } \\
\text { House }\end{array}$ & Fox Pro & Fox Pro & DOS & $\begin{array}{l}\text { Stand } \\
\text { Alone }\end{array}$ & Live \\
\hline
\end{tabular}




\begin{tabular}{|c|c|c|c|c|c|c|c|c|c|c|}
\hline 66 & $\begin{array}{l}\text { Bank and } \\
\text { ministries } \\
\text { Coordination } \\
\text { Application }\end{array}$ & $\begin{array}{l}\text { Maintains } \\
\text { Government } \\
\text { Accounts }\end{array}$ & $\begin{array}{l}\text { Decentralize } \\
\mathrm{d}\end{array}$ & $\begin{array}{l}\text { Single } \\
\text { User }\end{array}$ & $\begin{array}{l}\text { In } \\
\text { House }\end{array}$ & Fox Pro & Fox Pro & DOS & $\begin{array}{l}\text { Stand } \\
\text { Alone }\end{array}$ & Live \\
\hline 67 & $\begin{array}{ll}\text { Profit } & \text { Rates } \\
\text { Declaration } & \\
\text { Application } & \end{array}$ & $\begin{array}{l}\text { To declare the } \\
\text { rates on PLS } \\
\text { Deposits }\end{array}$ & $\begin{array}{l}\text { Decentralize } \\
\mathrm{d}\end{array}$ & $\begin{array}{l}\text { Single } \\
\text { User }\end{array}$ & $\begin{array}{l}\text { In } \\
\text { House }\end{array}$ & Visual FoxPro & $\begin{array}{l}\text { Visual } \\
\text { FoxPro }\end{array}$ & $\begin{array}{l}\text { Windows } \\
\text { (PC) }\end{array}$ & $\begin{array}{l}\text { Stand } \\
\text { Alone }\end{array}$ & Live \\
\hline 68 & $\begin{array}{l}\text { Retired Employees } \\
\text { Facilities System-II }\end{array}$ & $\begin{array}{l}\text { Calculation of } \\
\text { Medical Grant } \\
\text { on } \\
\text { Enhancement } \\
\text { of Pension }\end{array}$ & $\begin{array}{l}\text { Decentralize } \\
\mathrm{d}\end{array}$ & $\begin{array}{l}\text { Single } \\
\text { User }\end{array}$ & $\begin{array}{l}\text { In } \\
\text { House }\end{array}$ & Visual FoxPro & $\begin{array}{l}\text { Visual } \\
\text { FoxPro }\end{array}$ & $\begin{array}{l}\text { Windows } \\
(\mathrm{PC})\end{array}$ & $\begin{array}{l}\text { Stand } \\
\text { Alone }\end{array}$ & Live \\
\hline 69 & $\begin{array}{l}\text { Adjusted } \\
\text { Statement of } \\
\text { Financial Affairs }\end{array}$ & $\begin{array}{l}\text { Adjusted } \\
\text { statement of } \\
\text { affairs of Bank }\end{array}$ & $\begin{array}{l}\text { Decentralize } \\
\mathrm{d}\end{array}$ & $\begin{array}{l}\text { Single } \\
\text { User }\end{array}$ & $\begin{array}{l}\text { In } \\
\text { House }\end{array}$ & FoxPro & FoxPro & DOS & $\begin{array}{l}\text { Stand } \\
\text { Alone }\end{array}$ & Live \\
\hline 70 & WST PC & $\begin{array}{l}\text { Collection of } \\
\text { Soft WST data }\end{array}$ & $\begin{array}{l}\text { Decentralize } \\
\mathrm{d}\end{array}$ & $\begin{array}{l}\text { Single } \\
\text { User }\end{array}$ & $\begin{array}{l}\text { In } \\
\text { House }\end{array}$ & FoxPro & FoxPro & DOS & $\begin{array}{l}\text { Stand } \\
\text { Alone }\end{array}$ & Live \\
\hline 71 & $\begin{array}{l}\text { Travelling } \\
\text { Allowances } \\
\text { Monitoring System }\end{array}$ & $\begin{array}{l}\text { maintenance of } \\
\text { travelling } \\
\text { expenses / } \\
\text { foreign trips }\end{array}$ & $\begin{array}{l}\text { Decentralize } \\
\mathrm{d}\end{array}$ & $\begin{array}{l}\text { Multi } \\
\text { user }\end{array}$ & $\begin{array}{l}\text { In } \\
\text { House }\end{array}$ & C \#, ASP .net & $\begin{array}{l}\text { SQL } \\
\text { Server }\end{array}$ & $\begin{array}{l}\text { Windows } \\
\text { (PC) }\end{array}$ & On Line & $\begin{array}{l}\text { un } \\
\text { operat } \\
\text { ional }\end{array}$ \\
\hline 72 & Chart of Accounts & $\begin{array}{l}\text { Consolidation } \\
\text { of profit and } \\
\text { loss, statement } \\
\text { of affairs and } \\
\text { other } \\
\text { subsidiary } \\
\text { statements at } \\
\text { year end }\end{array}$ & $\begin{array}{l}\text { Decentralize } \\
\mathrm{d}\end{array}$ & $\begin{array}{l}\text { Multi } \\
\text { user }\end{array}$ & $\begin{array}{l}\text { In } \\
\text { House }\end{array}$ & FoxPro & FoxPro & DOS & $\begin{array}{l}\text { Stand } \\
\text { Alone }\end{array}$ & Live \\
\hline 73 & $\begin{array}{l}\text { Real Time Gross } \\
\text { Settlement (RTGS) }\end{array}$ & $\begin{array}{l}\text { Real Time } \\
\text { funds transfer } \\
\text { between banks } \\
\text { through } \\
\text { treasuries }\end{array}$ & $\begin{array}{l}\text { Decentralize } \\
\text { d }\end{array}$ & $\begin{array}{l}\text { Multi } \\
\text { user }\end{array}$ & $\begin{array}{l}\text { In } \\
\text { House }\end{array}$ & FoxPro & FoxPro & DOS & $\begin{array}{l}\text { Stand } \\
\text { Alone }\end{array}$ & Live \\
\hline 74 & $\begin{array}{l}\text { Assets and } \\
\text { LiabilitiesCommitt } \\
\text { ee (ALCO) }\end{array}$ & $\begin{array}{l}\text { Assets and } \\
\text { liabilities entry } \\
\text { system }\end{array}$ & $\begin{array}{l}\text { Decentralize } \\
\mathrm{d}\end{array}$ & $\begin{array}{l}\text { Multi } \\
\text { user }\end{array}$ & $\begin{array}{l}\text { In } \\
\text { House }\end{array}$ & FoxPro & FoxPro & DOS & $\begin{array}{l}\text { Stand } \\
\text { Alone }\end{array}$ & Live \\
\hline 75 & $\begin{array}{l}\text { Monthly Budget } \\
\text { System }\end{array}$ & $\begin{array}{l}\text { preparation of } \\
\text { monthly } \\
\text { budget }\end{array}$ & $\begin{array}{l}\text { Decentralize } \\
\mathrm{d}\end{array}$ & $\begin{array}{l}\text { Multi } \\
\text { user }\end{array}$ & $\begin{array}{l}\text { In } \\
\text { House }\end{array}$ & FoxPro & FoxPro & DOS & $\begin{array}{l}\text { Stand } \\
\text { Alone }\end{array}$ & $\begin{array}{l}\text { un } \\
\text { operat } \\
\text { ional }\end{array}$ \\
\hline 76 & $\begin{array}{l}\text { Monthly Statement } \\
\text { of Liabilities and } \\
\text { Assets }\end{array}$ & $\begin{array}{l}\text { Assets and } \\
\text { liabilities entry } \\
\text { system and } \\
\text { reports } \\
\text { generation }\end{array}$ & $\begin{array}{l}\text { Decentralize } \\
\mathrm{d}\end{array}$ & $\begin{array}{l}\text { Multi } \\
\text { user }\end{array}$ & $\begin{array}{l}\text { In } \\
\text { House }\end{array}$ & FoxPro & FoxPro & DOS & $\begin{array}{l}\text { Stand } \\
\text { Alone }\end{array}$ & Live \\
\hline 77 & $\begin{array}{l}\text { Equity Portfolio } \\
\text { Management } \\
\text { System }\end{array}$ & $\begin{array}{l}\text { Management } \\
\text { of equity } \\
\text { portfolio of } \\
\text { Bank }\end{array}$ & $\begin{array}{l}\text { Decentralize } \\
\text { d }\end{array}$ & $\begin{array}{l}\text { Single } \\
\text { User }\end{array}$ & $\begin{array}{l}\text { In } \\
\text { House }\end{array}$ & FoxPro & FoxPro & DOS & $\begin{array}{l}\text { Stand } \\
\text { Alone }\end{array}$ & Live \\
\hline 78 & $\begin{array}{l}\text { General Ledger } \\
\text { and Subsidiary } \\
\text { Accounts }\end{array}$ & $\begin{array}{l}\text { Maintain the } \\
\text { GL of finance } \\
\text { group }\end{array}$ & $\begin{array}{l}\text { Decentralize } \\
\text { d }\end{array}$ & $\begin{array}{l}\text { Single } \\
\text { User }\end{array}$ & $\begin{array}{l}\text { In } \\
\text { House }\end{array}$ & FoxPro & FoxPro & DOS & $\begin{array}{l}\text { Stand } \\
\text { Alone }\end{array}$ & Live \\
\hline 79 & $\begin{array}{l}\text { Monthly Profit and } \\
\text { Loss statement } \\
\text { application }\end{array}$ & $\begin{array}{lr}\text { Monthly } & \text { Profit } \\
\text { and } & \text { Loss } \\
\text { Positions } & \end{array}$ & $\begin{array}{l}\text { Decentralize } \\
\mathrm{d}\end{array}$ & $\begin{array}{l}\text { Single } \\
\text { User }\end{array}$ & $\begin{array}{l}\text { In } \\
\text { House }\end{array}$ & FoxPro & FoxPro & DOS & $\begin{array}{l}\text { Stand } \\
\text { Alone }\end{array}$ & Live \\
\hline 80 & $\begin{array}{l}\text { Mergers \& } \\
\text { Acquisitions } \\
\text { Management }\end{array}$ & $\begin{array}{l}\text { Management } \\
\text { of e-CIB, GL } \\
\text { and Loans } \\
\text { received } \\
\text { through } \\
\text { mergers and } \\
\text { acquisitions }\end{array}$ & $\begin{array}{l}\text { Decentralize } \\
\mathrm{d}\end{array}$ & $\begin{array}{l}\text { Single } \\
\text { User }\end{array}$ & $\begin{array}{l}\text { In } \\
\text { House }\end{array}$ & FoxPro & FoxPro & DOS & $\begin{array}{l}\text { Stand } \\
\text { Alone }\end{array}$ & $\begin{array}{l}\text { un } \\
\text { operat } \\
\text { ional }\end{array}$ \\
\hline 81 & ATM/Debit Card & $\begin{array}{l}\text { ATM/Debit } \\
\text { Card Host, } \\
\text { Switch } \\
\text { Interface (1- } \\
\text { Link/Orix), } \\
\text { Interface for } \\
\text { General } \\
\text { Banking and } \\
\text { Reporting. }\end{array}$ & Centralized & $\begin{array}{l}\text { Multi } \\
\text { User }\end{array}$ & Both & COBOL, RPG, CL & DB2 & OS/400 & On-line & Live \\
\hline 82 & $\begin{array}{l}\text { ATM } \\
\text { Reconciliation } \\
\text { System }\end{array}$ & $\begin{array}{l}\text { To Support } \\
\text { ATM } \\
\text { Settlement } \\
\text { Dept in } \\
\text { Reconciliation }\end{array}$ & Centralized & $\begin{array}{l}\text { Multi } \\
\text { User }\end{array}$ & Both & $\begin{array}{l}\text { Oracle } \\
\text { Developer 6i }\end{array}$ & $\begin{array}{l}\text { Oracle } \\
10 \mathrm{~g}\end{array}$ & LAN & On-line & Live \\
\hline
\end{tabular}


Reasons for Low Information Technology Growth in Master Bank: Main factors responsible for slow IT diffusion in Master Bank are as follows,

Supply Side Constraints: The Master Bank is affected by Supply-side constraints which include the state monopoly of telecommunications and the existence of regulations that prevent the private sector from participating in the sector. The telecommunication companies and the software developers are fewer in Pakistan therefore, the Banks in Pakistan heavily rely upon the outsourced systems, purchased from abroad. Also contributory is the lack of resources to augment investment in telecommunications sector sometimes due to the high level of indebtedness of the local operator. For speedy and efficient E Banking services, a strong network infrastructure is a must which is lacking in Master Bank and its only E Banking facility i.e ATM is frequently disconnected from source resulting in long down times.

High Population Growth Rates: Population growth rate in Pakistan is high as compared to its productive capacity. It is projected that in year 2011, the rate will be 24.81 births / 1000 population. This is giving rise to increased deposit base of Master Bank. As the number of customers is growing, Master bank of Pakistan is focusing on procuring more depositors rather than focusing on facilitating existing customers and cost reduction by infusion of technology in Banking services and operations.

Low Investment Efficiency and High Installation Costs: Installation costs per line in Pakistan are on higher side than the industry average which is above US\$ 4,500 per line. The huge installation costs are partly due to the large rural areas that have to be covered. These large costs are reflected in high subscription charges.

Civil Disturbances and Political Instability: A factor accounting for the lack of development in Information technology in Master Bank is the absence of political stability in Pakistan. Also, the Master Bank has existence in the areas which are mainly affected by terrorism. Complete system Automation in the terrorism affected are is very difficult as the IT personnel are unable to visit the areas and the telecommunication infrastructure is difficult to be installed.

Absence of Adequate Communication Network Infrastructures: Generally, in Banks providing E Banking services, arrange for appropriate and sufficient communication equipments. In Master Bank although, two parallel communication systems are installed for its 384 online branches, i.e optic fiber and DSL as backup communication connection.

Lack Of Government Interest And Support: Master Bank is mainly owned by Government of Pakistan. The government is not taking much interest in automation of the Baking services and operations as the Master Bank being Government owned holds major deposit and business of the government. The Master bank has its monopoly in many banking services which other foreign and domestic banks are not offering like government transactions involving huge funds, revenue collection etc. This leads to lack of competitiveness in Master Bank causing in low interest in introducing technology enabled customized banking services. Also, being a government owned organization, it faces restrictions and fulfillment of certain minimum requirements.

Technical And Management Support For Internet Connection: The Master bank being an old bank and facing restrictions on external hiring has low quality of human resource. The human resource is very critical factor in the services industry like Banking, but the Master bank is far behind the domestic and foreign banks as its HR is not well equipped with the changing business environment. They are not qualified enough to handle the problems associated with web based and electronic banking products. Only $20 \%$ of Master Banks' human resource is computer literate -a big question mark is. As Master Bank uses a weak communication infrastructure, technical problems occur quite often increasing the system down time. 
Regulators' guidelines: The State Bank of Pakistan has given security policy for E Banking transactions, mobile banking, credit cards and branchless banking. To coop with rapidly changing requirements of regulator, it is becoming difficult for Master Bank to convert its system into fully automated one.

Lack Of Effective Coordination Between Groups: Master Bank being a huge organization consists of multilayer hierarchy and groups. There is lack of communication and coordination between groups and offices mainly due to least use of IT for official communication. This causes slow implementation of IT projects in Master Bank.

High Equipment Costs: The Branch automation requires installation of sophisticated equipments to handle multi user applications and deliver efficient output at the same time. In Pakistan, these are not produced locally and Banks have to rely on imported equipment which involves heavy payment in foreign exchange which hinders the automation of Master Bank.

Internal Issues: As mentioned earlier, Master Bank has less qualified staff, lack of proper training, improper work distribution, rapid changes in top level management and political influences. Although, the Master Bank issues IT user manual from time to time, these are not properly circulated to concerned employees to update their knowledge and practices which results in slow rate of IT projects implementation. Also, the employees show maximum level of resistance for every change in organization mainly in field of IT.

Large Branch Network: The Master bank consists of largest branch network in Pakistan having 1256 branches all over country having appearance in far flung areas where no other domestic or foreign bank exists. It is very difficult to converted whole network into real time online overnight. This work must have to be done in chunks. As it appears in the areas where poor social and communication infrastructure prevails, even no communication service provider company exists, full automation of complete branch network is not possible.

Lack of Interest by Senior Management: Achieving success in financial sector in today's volatile and uncertain business environment requires innovation and proven expertise. The senior management of Master Bank is still unable to understand the intensity of need of technology oriented customized services. Therefore, implementation of IT projects is emphasized less by them as compared to deposit procurement and reduction in classified loans portfolio. It serves as a main cause of slow IT growth rate in Master Bank.

Non Existence Of Marketing Department In Master Bank: Being a Government owned entity, the master Bank still have no separate marketing group to plan and launch new Banking products manly due to monopolistic control over some Banking activities. Also, as State bank of Pakistan requires launch of proper software for monitoring and management of every banking product, Master Bank is reluctant to launch new It oriented consumer products which indirectly hinders growth of IT in Master Bank.

Future of it in Master Bank: The upcoming IT projects in Master Bank are as follows;

- Branches are in process of shifting to the higher bandwidth under a contract with PTCL that will increase the efficiency and connectivity.

- Maximum number of Branches will be made on line by December 2011.

- Electronic Remittance system is already working.

- Introduction of HR centralization system will bring a revolution in the HR data maintenance of the Master Bank.

- The most important aspect is launching and implementation of CORE BANKING SYSTEM. This system is purchased from the most leading firm of the world Fidelity Intl. It is the highest funded IT project of banking history in Pakistan. It will make Master Bank a one window operation bank. Online money transfers and payments from any branch will be possible. The system has already been successfully implemented in a branch in Karachi and two branches are running in trial. Shortly, its implementation will start in other big cities as well. Shortly implementation will start in Lahore and gradually in whole country. 
- Another revolution is the launch of mobile banking. The system is ready and on hold as it is in the process of obtaining approval from State Bank of Pakistan.

- The Master bank has marked a historic milestone by launching online connectivity for network expansion project. For this, the bank has made alliance with PTCL to fully automate its 1256 branches. The project will provide the bank a platform for the roadmap towards a paperless banking environment.

- So very soon the Master bank will lead not only in highest profit rather also in information technology facilities for customers.

Challenges and Risks: The major challenges facing ICT adoption can therefore be summarized under the following categories:

Sustainable Wired and Wireless Networks: The Master Bank needs to make agency arrangements with country's' best communication network service providers in order to capture the major share of electronic banking in market.

Cost of Connection: The connection costs are normally very high, therefore the Master bank must sort out different ways to lower the connection costs. For this the Master bank must enter into agreement with service provider and negotiate the cost of connection. A major barrier in IT diffusion is the limited availability of communication infrastructures in developing countries. Existing communication networks are available only in urban areas, offering poor services in contrast with the ultra high-speed and modern electronic banking systems operational in foreign banks operating in Pakistan. So, modernizing existing communication channels and infrastructures as well as establishing new ones in remote areas is thus the key issues.

E-Security Issues: As the use of electronic and technology based services increases, question of transaction security rises. To coop up with the issue, the Master Bank must deploy a strong communication network and compliance of State Bank of Pakistan E security policy guidelines. Also, the user IDs and authorities must be assigned in such a way as to avoid access of system by UN authorized persons.

Political Instability - Policy Inconsistencies: In Pakistan, political and social unrest is prevailing mainly due to terrorism and strategic and demographic importance of Pakistan in the region. In this part of south Asia, political government hardly completes its constitutional tenure and changes occur rapidly. Every coming team in government immediately terminates the policies announced by previous government and imposes new programs and policies. This phenomenon has become a major challenge for Master Bank in implementation of its long term plans. As Government of Pakistan is a major shareholder of Master Bank, it is always influenced by the change in government.

Lack Of Effective Coordination: In Master Bank, due to formation of large number of groups, it faces lack of coordination among various groups. This is serving as obstacle in the way of smooth and prompt implementation of IT projects in Master Bank.

\section{Risk Management Challenges}

Adaptation to Technology issues: The rapid changes in technological and customer service innovation in ebanking are unprecedented. This magnifies the challenges to the Master Banks' management to ensure that adequate strategic assessment, risk analysis and securities reviews and revaluations are conducted prior to implementing new e-banking applications. The Human resource who is the first to encounter with the automated systems is reluctant to accept any change in organization due to lack of qualifications, computer literacy and initiative, so Master Bank before becoming fully automated must have well thought plans to address the issues of technology adoption.

Outsourcing issue: E-banking increases banks' dependence on technology, thereby increasing the technical complexity of many operational and informational matters. It also gives rise to E-security issues and furthering a trend towards partnerships, alliances and outsourcing arrangements with unregulated third 
parties. Managing the package of risks associated to the unregulated partners and third parties and security issues are the first and foremost to be addressed.

Increased legal and reputational risks: $\mathrm{E}$ banking brings with itself security and swift delivery of service issues. The customers using E banking services are mostly concerned with the time saving linked with it. A strong communication network guarantees this advantage to the customers. If a bank fails to provide so, it is bound to face reputational and operational risks in contrast with its long term profit maximization objectives. The e banking exposes a Bank to legal risks as well due to poor security policies governing the E banking channels.

E-security issue: E banking is usually web based and it is an open network accessible from anywhere in the world by unknown persons, at unknown locations and through wireless devices. Therefore, it is incumbent of challenges on security controls, customer identification, data control standards, audit procedures, and customer privacy requirements. Every country depending heavily on E Banking delivery channels is exposed to the same risk. Since 1999, Brazil has faced $418 \%$ increase in e security issues; Korea has experienced 932\% rise and Japan has evidenced over $1000 \%$ increase in malicious electronic banking transactions (Tom Glaessner et al, 2003).More than 57\% of all hack attacks in 2002 were attempted against the Banking sector (Tom Glaessner et al, 2003).

Policy Implications and Some Suggesting Thoughts: As Master Bank is planning to convert its whole network into fully automated in future it must formulate its policies in such a way to achieve ultimate strategic goals of the customer satisfaction maximization, increase in Banks' net worth, reduction in costs and attaining sustainable competitive advantage.

- The Master Bank must establish a comprehensive security control process. A strong e banking customer's authentication and identification policy must be in place. The Master Bank must build up a comprehensive e-security public policy framework. There is a strong public interest case for government to regulate the e-financial transactions as Master Bank is being owned by government. The Internetbased transactions require high security measures for which private solutions and troubleshooting may not be sufficient. The reach of the internet technologies imply that financial services are becoming global and boundary-less. Therefore, mitigating e security risks requires vigorous efforts to consolidate a combined auctioned plan by participants, regulators, and law enforcements.

- The Human resource department in coordination with IT group must assign appropriate duties to employees handling the e banking applications to avoid unauthorized access to the system.

- The Master Bank must establish clear audit tests and trails for every e-banking transaction. A set of rules must be designed for accountability for e-banking transactions. In Master Bank, for successful implementation of its IT delivery channels, centralized-back office must be established to free staff time in sales and services areas and for consolidation of process in harmony across the Bank.

- For loans automation, the Master Bank must develop automated credit authorization system by introducing an appropriate credit scoring and cash- flow based financing system for reduction of operating costs, maintaining asset quality, and increasing consumers' profitability. It will enable the Bank to take lending decisions without obtaining the projected financials, credit reports and hard to get collateral valuation reports.

- The Master Bank must have in place a comprehensive due diligence and management oversight policy for outsourcing alliances and other unregulated third-party partnerships. It must undertake strategic collaborations within the players of industry particularly in the research and development areas (software development and securities), promotional and awareness activities.

- The management of Master Bank must integrate cross-border e-banking risks into the bank's overall strategic risk management policy. For avoidance of legal and reputational risks associated with E Banking, the Bank must appropriately disclose its E Banking services to its customers and regulators. It must ensure the privacy of customer information and transactions through strong communication infrastructure. Different contingency plans must be in place to ensure the availability of E Banking services. The Law and order situation in country is very critical and to encounter this issue the Bank must develop an appropriate incident response policy. Also, again the duties must be appropriately segregated so as to avoid illegal and unidentified access to electronic banking system. 
- The Master Bank must hold the ability to adopt global technology according to local requirements: An adequate level of communication and information infrastructure and human capability building are required before adopting the global technology to meet local requirements.

\section{Conclusion}

Banking milieu has become exceedingly competitive and to survive and grow in the changing market environment, it has become imperative to successful banks to opt latest technologies. It is professed as an enabling resource that can facilitate in developing more flexible organization that can retort quickly to the dynamics of fast growing markets. The paradigm shift is result of continuous emergence of new foreign and domestic banks in Pakistan. Accordingly, to keep up with latest trends and fashions in banking industry, Master Bank is slowly shifting from traditional banking to high-tech banking. It is in the process of elaborating its existing IT setup by embarking on centralization of online branches. For this, it has formulated IT plans and business support approaches to retain customers and improve IT support services. The Master Bank is facing key issues of lack of regulatory frameworks, customers' trust, e security issues, privacy standards, customers identification and transaction authentication techniques etc. to implement e- Banking projects. By concluding well thought out policies and regulatory frameworks, keeping in view the principles of Basel Accord and using online banking as new delivery tools Master Bank will be soon conquering the skies of success as financial super market and the Bank of first choice in Pakistan.

\section{References}

Aladwani, A. M. (2000). IS project characteristics and performance: a Kuwaiti illustration. Journal of Global Information Management, 8(2), 50-7.

Aladwani, A. M. (2001). IT planning effectiveness in a developing country. Journal of Global Information Management, 4(3), 51-65

Al-Otaibi, S. B. (1992). Linking strategy, information technology and performance in financial service organization: An empirical investigation in the Gulf Cooperation Council Countries IMCS 14,5 482(Saudi Arabia, Kuwait, Bahrain, Qatar, Oman, United Arab Emirates), unpublished DBA dissertation, Southern Illinois University at Carbondale, Carbondale, IL.

AL-Rawashdeh, B. S., Abu-Errub, A. M., Areiqat, A. Y. \& Dbbaghieh, M. (2012). Information Technology Role in Reducing E-Banking Services Risk in Jordanian Banking Sector. Journal of Computer Science, 3, 374381.

Al-Tawil, K. M. (2001). The internet in Saudi Arabia. Telecommunications Policy, 25(8/9), 625-32.

Athanassopoulos, A. D. \& Iliakopoulos, A. (2003). Modeling customer satisfaction in telecommunications: Assessing the effects of multiple transactions points on the perceived overall performance of the provider. Production and Operation Management, 12, 224-245.

Andersen, A. (2001). Dubai makes a bid for e-business. International Tax Review, 12(1), 47-9.

Awamleh, R., Evans, J. \& Mahate, A. (2003). Internet banking in emerging markets, The case of Jordan ?A note. Journal of Internet Banking and Commerce, 8(1).

Berger, A. N. (2003). The economic effects of technological progress: evidence from the banking industry. Journal of Money, Credit, Banking, 35(2), 141-176.

Brown, I. \& Molla, A. (2005). Determinants of Internet and cell phone banking adoption in South Africa. Journal of $g$ and Commerce, 10(1).

Brynjolfsson, E. \& Hitt, L. (2000). Beyond Computation: Information Technology, OrganizationalTransformation and Business Performance. Journal of Economic Perspectives, 14(4).

Burn, J. M., Tye, E. M. W. N. \& Ma, L. C. K. (1995). Paradigm shift - cultural implications for deployment of IS professionals. Journal of Global Information Management, 3(2), 18-28.

Burn, J. M. \& Loch, K. D. (2001). The societal impact of the world wide web - key issues for the 21st century. Information Resources Management Journal, 14(4), 4-14.

Chotani, P. Y., Siva, P. \& Narayanan, L. (2004). From <http:// www.crmzday.com, Retrieved 12-05-2011

Chou, H. W. \& Jou, S. B. (1999). MIS key issues in Taiwan's enterprises. International Journal of Information Management, 19(5), 369-87.

Cohen, J. E. (2006). Citizen satisfaction with contacting government on the internet. Information Policy, 11, 5165. 
Corvoisier, S. \& Gropp, R. (2009). Contestability, Technology and Banking. Sandrine Corvoisier and Reint Gropp.

Dasgupta, S. (1999). Determinants of information technology adoption: an extension of existing models to firms in a developing country. Journal of Global Information Management, 7(3), 30-40.

Diniz, E. (1998). Web Banking in USA. Journal of Internet Banking and Commerce, 3(2).

Domegan, C. (1995). Information technology and customer service, unpublished DPhil thesis, University College Galway.

Dos Santos, B. \& Pfeffers, D. (1993). Firm level performance effects: a framework for information technology evaluation research, in Banker, R.D., Kauffman, R.J. and Mahmood, M. (Eds), Strategic Information Technology Management: Perspectives on Organizational Growth and Competitive Advantage, Idea Group Publishing, Harrisburg, PA.

D-Subbarao, G. (2009). Reserve Bank of India on May 18, 2009 at the Banking Technology Awards 2008 of the Institute for Development \& Research in Banking Technology, Hyderabad.

Economides, N. \& Salop, S. (1992). Competition and integration among complements, and network market structure. The Journal of Industrial Economics, XL(1), 105-123

Eisenhardt, K. M. (1989). Building theories from case study research. Academy of Management Review, 14(4), 532-582.

Ezehoha, A. E. (2005). Regulating Internet banking in Nigeria: Problems and challenges ?Part 1. Journal of Internet Banking and Commerce, 10(3).

Farrell, J. \& Saloner, G. (1985). Standardization, compatibility and innovation. RANDJournal of Economics, 16 (1), 70-83.

Garau, C. (2002). Online banking in transition economies: The implementation and development of online banking systems in Romania. International Journal of Bank Marketing, 20(6), 285-296.

Goi, C. L. (2005). E-Banking in Malaysia: Opportunity and challenges. Journal of Internet Banking and Commerce, 10(3).

Gordon, T. J. (2002). And who asked your opinion? Information World Review, 178, 14.

Hallam, S. (2001). New disruptive technologies: the information professional's role. BusinessInformation Review, 18(3), 21-8.

Harold, B. \& Jeff, L. (1995). Don't Let Technology Pass You By', ABA Banking Journal, Box 986, Omaha, NE, p.73

Harwood, P. (2001). You are the weakest link: goodbye: serving the information hungrycorporate end user, Online Information 2001, Proceedings of the International OnlineMeeting, London, 4-6 December, 2001, 67-70.

Heckman, R. (1998). Planning to solve the 'skill problem' in the virtual information managementorganization. International Journal of Information Management, 18(1), 3-16.

Hernan, E., Riquelme, K. A. \& Mekkaoui, R. E. R. (2009). Internet Banking Customer Satisfaction and Online Service Attributes. Journal of Internet Banking and Commerce, 14(2).

Higgins, G. M. Jr (2001). If you build it, will they come? A study of the effect of systems structure on the institutionalization of knowledge management within organizations, unpublished $\mathrm{PhD}$ dissertation, University of Georgia, Athens, GA

Ho, S. J. \& Mallick, S. K. (2010). The Impact of Information Technology on the Banking Industry: Theory and Empirics. Journal of the Operational Research Society, 2(1).

Houissa, A. (2000). The Internet predicament in the Middle East and North Africa: connectivity, access and censorship. Journal of Librarianship and Information Science, 32(2), 56-63.

Ibrahim, E. E., Joseph, M. \& Ibeh, K. I. N. (2006). Customers' perception of electronic service delivery in the UK retail banking sector. International Journal of Bank Marketing, 24(7), 475-493.

ITU Basic ICT Statistics. (2006). ICT Statistics, World Telecommunications Indicators Database 9th Edition, International Telecommunication Union (ITU), Retrieved 12 June, 2011, from http://www.itu.int/ITU-D/ict/statistics/ict/index.html.

Jasimuddin, S. M. (2001). Saudi Arabian banks on the Web. Journal of Internet Banking and Commerce, 6(1).

Jayawardhena, C. \& Foley, P. (2000). Changes in the banking sector - the case of internet banking in the UK. Internet Research: Electronic Networking Applications and Policy, 10(1), 19-30.

Joseph, M. \& Stone, G. (2003). An empirical evaluation of US bank customers' perceptions of the impact of technology on services delivery in the banking sector. International Journal of Retail \& Distribution Management, 31(4), 190-202. 
Ju, T. L., Chen, S., Li, C. \& Lee, T. (2005). A strategic contingency model for technology alliance. Industrial Management \& Data Systems, 105(5), 623-44.

Katz, M. \& Shapiro, C. (1985). Network externalities, competition, and compatibility. The American Economic Review, 75(3), 424-440.

Kozak, S. (2005). The role of information technology in the profit and cost efficiency improvements of the banking sector. Journal of Academy of Business and Economics, 2(1).

Liao, Z., Landry-Jr, R. \& Cheung, M. T. (2003). Banking Information Success Acceptance: An Empirical Evaluation. The Review of Business Information Systems, 3(1).

Lin, F., Sheng, O. \& Wu, S. (2005). An integrated framework for eChain bank accounting systems. Industrial Management \& Data Systems, 105(3), 291-306.

Mabberly, J. (1996). The Price Waterhouse Guide to Activity-based Costing for Financial Institutions, Irwin Books, Burr Ridge, IL.

Mbarika, V. A. W. \& Okoli, C. (2003). A framework for assessing e-Commerce in Sub-Saharan Africa. Journal of Global Information Technology Management, 6(3) 44-66.

$\mathrm{Mu}$, Y. (2003). E-Banking: Status, Trends, Challenges and Policy Issues, conference on The Development and Supervision of E-banking, Shanghai, Nov. 24-26

Neptune. (2006). Equinox Universal Banking Solution, Neptunes Software Plc., Retrieved 12 April 2011, from http://www.neptunesoftwareplc.com/eqinox_universal_banking_solution.htm.

Orlikowski, W. J. \& Baroudi, J. J. (1991). Studying information technology in organizations: research approaches and assumptions. Information Systems Research, 2(1), 1-28.

Permual, S. A. (2006). Effective method of security measures in virtual banking. Journal of Internet Banking and Commerce, 11(1).

Perreault, M., Pawliuk, N., Veilleux, R. \& Rousseau, M. (2006). Qualitative assessment of mental health service satisfaction: strengths and limitations of a self-administered procedure. Community Mental Health Journal, 42, 233-241.

Rao, N. V. M., Singh, P. \& Maheshwari, N. (2005). A framework for evaluating e-business models and productivity analysis for banking sector in India. Journal of Internet Banking and Commerce, 10(2).

Remenyi, D. (1991). An Introduction to Strategic Planning, NCC Blackwell, London.

Richard, B. \& Alemayehu, M. (2006). Developing E-banking Capabilities in a Ghanaian Bank: Preliminary Lessons. Journal of Internet Banking and Commerce, 11(2), 213-34.

Rotchanakitumnuai, S. \& Speece, M. (2003). Barriers to Internet banking adoption: A qualitative study among customers in Thailand. International Journal of Bank Marketing, 21(6/7), 312-323.

Singh, B. \& Malhotra, P. (2004). Adoption of Internet banking: An empirical investigation of Indian banking Sector. Journal of Internet Banking and Commerce, 9(2).

Shu, W. \& Strassmann, P. A. (2005). Does information technology provide banks with profit? Information and Management, 42(5), 781-787.

Slowinsky, J. (2000). Workforce literacy in an information age: policy recommendations for developing an equitable high-tech skills workforce. First Monday, 5(7).

Smith, J. M. \& Webster, L. (2000). The knowledge economy and SMEs: a survey of skills requirement. Business Information Review, 17(3), 138-46.

Stratigos, A. (2001). Knowledge management meets future information needs. Online, 25(1), 65-7.

Sun, R. (2010). Integrating Customer Relationship Management with Data Warehousing Technology - A Banking Industry Perspective. A dissertation submitted to Auckland University of Technology.

Szymankski, D. M. \& Henard, D. H. (2001). Customer satisfaction: a meta-analysis of the empirical evidence. Journal of the Academy Marketing Science, 29, 16-35.

Terblanche, N. S. (2006). The relationship between customer satisfaction and loyalty: An application of the American Customer Satisfaction Index in the South African fast food industry. Management Dynamics, $15,31-41$

Tom, G. (2003). Electronic security, risk mitigation in financial transactions. Journal of Internet Banking and Commerce, 11(1).

UNCTAD. (2002). E-Commerce And Development Report 2002", United Nations Conference on Trade and Development (UNCTAD), New York, Retrieved 9 July, 2011, from http://r0.unctad.org/ecommerce/ecommerce_en/edr02_en.htm.

Urgo, M. A. (1996). Computers and productivity: analysis of current literature and some significant issues. Business Information Review, 13(3), 195-8. 
Vijayan, P., Perumal, V. \& Shanmugam, B. (2005). Malaysia multimedia banking and technology acceptance theories. Journal of Internet Banking and Commerce, 10(1).

Welch, E. W., Hinnant, C. C. \& Moon, J. M. (2005). Linking citizen satisfaction with e-government and trust in government. Journal of Public Administration Research and Theory, 15, 371-391.

Westerback, L. K. (1999). Towards best practices for strategic information technology management. unpublished DPA dissertation, University of Southern California, LosAngeles, CA.

Williams, K. (2000). Are you ready for Internet banking? Strategic Finance, 81, 23 \& 82.

Woherem, E. W. (2000). Information Technology in the Nigerian Banking Industry, Spectrum, Ibadan

Yin, R. K. (1994). Case Study Research: Design and Methods, 2nd Edition. Thousand Oaks CA: Sage

Zhu, Z., Scheuermann, L. \& Babineaux, B. J. Jr (2004). Information network technology in the banking industry. Industrial Management \& Data Systems, 104(5), 409-17. 